Article

\title{
Multi-Criteria Decision-Making Method Using Heronian Mean Operators under a Bipolar Neutrosophic Environment
}

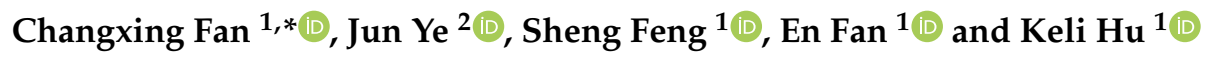 \\ 1 Department of Computer Science, Shaoxing University, 508 Huancheng West Road, Shaoxing 312000, China; \\ fengsheng_13@aliyun.com (S.F.); efan@usx.edu.cn (E.F.); ancimoon@gmail.com (K.H.) \\ 2 Department of Electrical and Information Engineering, Shaoxing University, 508 Huancheng West Road, \\ Shaoxing 312000, China; yehjun@aliyun.com \\ * Correspondence: fcxjszj@usx.edu.cn; Tel.: +86-575-8820-2669
}

Received: 23 November 2018; Accepted: 11 January 2019; Published: 17 January 2019

\begin{abstract}
In real applications, most decisions are fuzzy decisions, and the decision results mainly depend on the choice of aggregation operators. In order to aggregate information more scientifically and reasonably, the Heronian mean operator was studied in this paper. Considering the advantages and limitations of the Heronian mean (HM) operator, four Heronian mean operators for bipolar neutrosophic number (BNN) are proposed: the BNN generalized weighted HM (BNNGWHM) operator, the BNN improved generalized weighted HM (BNNIGWHM) operator, the BNN generalized weighted geometry HM (BNNGWGHM) operator, and the BNN improved generalized weighted geometry HM (BNNIGWGHM) operator. Then, their propositions were examined. Furthermore, two multi-criteria decision methods based on the proposed BNNIGWHM and BNNIGWGHM operator are introduced under a BNN environment. Lastly, the effectiveness of the new methods was verified with an example.
\end{abstract}

Keywords: bipolar neutrosophic number (BNN); BNN improved generalized weighted HM (BNNIGWHM) operator; BNN improved generalized weighted geometry HM (BNNIGWGHM) operator; decision-making

\section{Introduction}

In the real world, there is lots of uncertain information in science, technology, daily life, and so on. Particularly under the background of big data, the uncertainty of information is more complex and diverse. Now, how to make use of mathematical tools to deal with the uncertain information is an urgent problem for researchers. In order to describe uncertain information, Zadeh [1] put forward the concept of fuzzy sets. Considering the complexities and changes of uncertainty in the real environment, there was a certain limit on fuzzy sets to describe complex uncertainty; then, some extension theories [2-4] were put forward. Afterword, the neutrosophic set (NS) containing three neutrosophic components and the single-valued neutrosophic set were proposed by Smarandache [5], and the single-valued neutrosophic set was also mentioned by Wang and Smarandache [6]. Wang and Zhang [7] put forward an interval neutrosophic set (INS) theory. Furthermore, an $n$-value neutrosophic set [8] theory was proposed by Smarandache. The fuzzy set theory changed the binary view of people, but ignored the bipolarity of things. Under the background of big data, the confliction between data became more and more obvious. Traditional fuzzy sets could not do well in analyzing and handing uncertain information with incompatible bipolarity; this phenomenon was identified in 1994. For the first time, Zhang [9] introduced incompatible bipolarity into the fuzzy set theory, 
and put forward the bipolar fuzzy set (BFS). The founder of the fuzzy set theory, Zadeh, also affirmed that the bipolar fuzzy set theory was a breakthrough in traditional fuzzy set theory [10]. Then, Zemankova et al. [11] discussed a more generalized multipolar fuzzy problem, and pointed out that the multipolar fuzzy problem can be divided into multiple bipolar fuzzy problems. Chen et al. [12] studied m-polar fuzzy sets. Bosc and Pivert [13] introduced a study on fuzzy bipolar relational algebra. Manemaran and Chellappa [14] gave some applications of bipolar fuzzy groups. Zhou and Li [15] introduced some applications of bipolar fuzzy sets in semiring. Deli et al. [16] put forward a bipolar neutrosophic set (BNS), which can describe bipolar information. Later, some studies about BNS were put forward [17-20]. In this paper, we propose four Heronian mean operators for bipolar neutrosophic number (BNN). Compared with the literature [17-19], the HM operator can embody the interaction between attributes to avoid unreasonable situations in information aggregation. Compared with the literature [20], the Bonferroni mean (BM) aggregation operator not only neglects the relationship between each attribute and itself, but also considers the relationship between each attribute and other attributes repeatedly. However, the BM aggregation operator has large computational complexity, but the Heronian mean (HM) can overcome these two shortcomings.

The remaining sections are organized as follows: some related concepts are reviewed in Section 2. The four operators are defined and their properties are investigated in Section 3; these four operators are BNN generalized weighted HM (BNNGWHM), BNN improved generalized weighted HM (BNNIGWHM), BNN generalized weighted geometry HM (BNNGWGHM), and BNN improved generalized weighted geometry HM (BNNIGWGHM). Multi-criteria decision-making (MCDM) methods based on the BNNIGWHM and BNNIGWGHM operators are established in Section 4. A numerical example is provided and the effects of parameters $p$ and $q$ are analyzed in Section 5 . The conclusion of this paper is given in Section 6.

\section{Some Basic Concepts}

\subsection{BNN and Its Operational Laws}

Definition 1 [16]. Let $U=\left\{u_{1}, u_{2}, \ldots, u_{n}\right\}$ be a universe; a BNS $\Gamma$ in $U$ is defined as follows:

$$
\Gamma=\left\{\left\langle u, \alpha_{\Gamma}^{+}(u), \beta_{\Gamma}^{+}(u), \gamma_{\Gamma}^{+}(u), \alpha_{\Gamma}^{-}(u), \beta_{\Gamma}^{-}(u), \gamma_{\Gamma}^{-}(u)\right\rangle \mid u \in U\right\},
$$

in which $\alpha_{\Gamma}^{+}(u): U \rightarrow[0,1]$ means a truth-membership function, $\gamma_{\Gamma}^{+}(u): U \rightarrow[0,1]$ means a falsity-membership function and $\beta_{\Gamma}^{+}(u): U \rightarrow[0,1]$ means an indeterminacy-membership function, corresponding to a BNS $\Gamma$ and $\alpha_{\Gamma}^{-}(u), \gamma_{\Gamma}^{-}(u), \beta_{\Gamma}^{-}(u): U \rightarrow[-1,0]$ mean, respectively, the truth membership, false membership, and indeterminate membership to some implicit counter-property corresponding to a BNS $\Gamma$.

Definition 2 [16]. Let $U$ be a universe, and $\Gamma_{1}$ and $\Gamma_{2}$ be two BNSs.

$$
\begin{aligned}
& \Gamma_{1}=\left\{\left\langle u, \alpha_{\Gamma_{1}}^{+}(u), \beta_{\Gamma_{1}}^{+}(u), \gamma_{\Gamma_{1}}^{+}(u), \alpha_{\Gamma_{1}}^{-}(u), \beta_{\Gamma_{1}}^{-}(u), \gamma_{\Gamma_{1}}^{-}(u)\right\rangle \mid u \in U\right\}, \\
& \Gamma_{2}=\left\{\left\langle u, \alpha_{\Gamma_{2}}^{+}(u), \beta_{\Gamma_{2}}^{+}(u), \gamma_{\Gamma_{2}}^{+}(u), \alpha_{\Gamma_{2}}^{-}(u), \beta_{\Gamma_{2}}^{-}(u), \gamma_{\Gamma_{2}}^{-}(u)\right\rangle \mid u \in U\right\} .
\end{aligned}
$$

Then, the operations of $\Gamma_{1}$ and $\Gamma_{2}$ are defined as follows [16]:

(1) $\Gamma_{1} \subseteq \Gamma_{2}$, if and only if $\alpha_{\Gamma_{1}}^{+}(u) \leq \alpha_{\Gamma_{2}}^{+}(u), \beta_{\Gamma_{1}}^{+}(u) \geq \beta_{\Gamma_{2}}^{+}(u), \gamma_{\Gamma_{1}}^{+}(u) \geq \gamma_{\Gamma_{2}}^{+}(u)$, and $\alpha_{\Gamma_{1}}^{-}(u) \geq$ $\alpha_{\Gamma_{2}}^{-}(u), \beta_{\Gamma_{1}}^{-}(u) \leq \beta_{\Gamma_{2}}^{-}(u), \gamma_{\Gamma_{1}}^{-}(u) \leq \gamma_{\Gamma_{2}}^{-}(u)$;

(2) $\Gamma_{1}=\Gamma_{2}$, if and only if $\alpha_{\Gamma_{1}}^{+}(u)=\alpha_{\Gamma_{2}}^{+}(u), \beta_{\Gamma_{1}}^{+}(u)=\beta_{\Gamma_{2}}^{+}(u), \gamma_{\Gamma_{1}}^{+}(u)=\gamma_{\Gamma_{2}}^{+}(u)$, and $\alpha_{\Gamma_{1}}^{-}(u)=$ $\alpha_{\Gamma_{2}}^{-}(u), \beta_{\Gamma_{1}}^{-}(u)=\beta_{\Gamma_{2}}^{-}(u), \gamma_{\Gamma_{1}}^{-}(u)=\gamma_{\Gamma_{2}}^{-}(u)$; 
(3) $\Gamma_{1} \cup \Gamma_{2}=\left\{\left\langle u, \max \left(\alpha_{\Gamma_{1}}^{+}(u), \alpha_{\Gamma_{2}}^{+}(u)\right), \frac{\beta_{\Gamma_{1}}^{+}(u)+\beta_{\Gamma_{2}}^{+}(u)}{2}, \min \left(\gamma_{\Gamma_{1}}^{+}(u), \gamma_{\Gamma_{2}}^{+}(u)\right),\right\rangle \mid u \in U\right\}$;

$$
\begin{aligned}
& \min \left(\alpha_{\Gamma_{1}}^{-}(u), \alpha_{\Gamma_{2}}^{-}(u)\right), \frac{\beta_{\Gamma_{1}}^{-}(u)+\beta_{\Gamma_{2}}^{-}(u)}{2}, \max \left(\gamma_{\Gamma_{1}}^{-}(u), \gamma_{\Gamma_{2}}^{-}(u)\right) \\
& \text { (4) } \Gamma_{1} \cap \Gamma_{2}=\left\{\left\langle\begin{array}{r}
\left.\left.u, \min \left(\alpha_{\Gamma_{1}}^{+}(u), \alpha_{\Gamma_{2}}^{+}(u)\right), \frac{\beta_{\Gamma_{1}}^{+}(u)+\beta_{\Gamma_{2}}^{+}(u)}{2}, \max \left(\gamma_{\Gamma_{1}}^{+}(u), \gamma_{\Gamma_{2}}^{+}(u)\right),\right\rangle \mid u \in U\right\} ; \\
\max \left(\alpha_{\Gamma_{1}}^{-}(u), \alpha_{\Gamma_{2}}^{-}(u)\right), \frac{\beta_{\Gamma_{1}}^{-}(u)+\beta_{\Gamma_{2}}^{-}(u)}{2}, \min \left(\gamma_{\Gamma_{1}}^{-}(u), \gamma_{\Gamma_{2}}^{-}(u)\right)
\end{array}\right.\right.
\end{aligned}
$$

For convenience, we denote a bipolar neutrosophic number (BNN) by $\tau=\left\langle\alpha_{\tau}^{+}, \beta_{\tau}^{+}, \gamma_{\tau}^{+}, \alpha_{\tau}^{-}, \beta_{\tau}^{-}, \gamma_{\tau}^{-}\right\rangle$.

Definition 3 [16]. Let $\tau_{1}$ and $\tau_{2}$ be two BNNs, $\tau_{1}=\left\langle\alpha_{\tau_{1}}^{+}, \beta_{\tau_{1}}^{+}, \gamma_{\tau_{1}}^{+}, \alpha_{\tau_{1}}^{-}, \beta_{\tau_{1}}^{-}, \gamma_{\tau_{1}}^{-}\right\rangle$and $\tau_{2}\langle=$ $\left.\alpha_{\tau_{2}}^{+}, \beta_{\tau_{2}}^{+}, \gamma_{\tau_{2}}^{+}, \alpha_{\tau_{2}}^{-}, \beta_{\tau_{2}}^{-}, \gamma_{\tau_{2}}^{-}\right\rangle$, and $\delta>0$; then, the operations for BNNs are defined as follows [16]:

$$
\begin{gathered}
\tau_{1} \oplus \tau_{2}=\left\langle\alpha_{\tau_{1}}^{+}+\alpha_{\tau_{2}}^{+}-\alpha_{\tau_{1}}^{+} \alpha_{\tau_{2}}^{+}, \beta_{\tau_{1}}^{+} \beta_{\tau_{2}}^{+} \gamma_{\tau_{1}}^{+} \gamma_{\tau_{2}}^{+}, \alpha_{\tau_{1}}^{-} \alpha_{\tau_{2}}^{-}-\left(-\beta_{\tau_{1}}^{-}-\beta_{\tau_{2}}^{-}-\beta_{\tau_{1}}^{-} \beta_{\tau_{2}}^{-}\right),-\left(-\gamma_{\tau_{1}}^{-}-\gamma_{\tau_{2}}^{-}-\gamma_{\tau_{1}}^{-} \gamma_{\tau_{2}}^{-}\right)\right\rangle ; \\
\tau_{1} \otimes \tau_{2}=\left\langle\alpha_{\tau_{1}}^{+} \alpha_{\tau_{2}}^{+}, \beta_{\tau_{1}}^{+}+\beta_{\tau_{2}}^{+}-\beta_{\tau_{1}}^{+} \beta_{\tau_{2}}^{+}, \gamma_{\tau_{1}}^{+}+\gamma_{\tau_{2}}^{+}-\gamma_{\tau_{1}}^{+} \gamma_{\tau_{2}}^{+},-\left(-\alpha_{\tau_{1}}^{-}-\alpha_{\tau_{2}}^{-}-\alpha_{\tau_{1}}^{-} \alpha_{\tau_{2}}^{-}\right),-\beta_{\tau_{1}}^{-} \beta_{\tau_{2}}^{-}-\gamma_{\tau_{1}}^{-} \gamma_{\tau_{2}}^{-}\right\rangle ; \\
\delta \tau_{1}=\left\langle 1-\left(1-\alpha_{\tau_{1}}^{+}\right)^{\delta},\left(\beta_{\tau_{1}}^{+}\right)^{\delta},\left(\gamma_{\tau_{1}}^{+}\right)^{\delta},-\left(-\alpha_{\tau_{1}}^{-}\right)^{\delta},-\left(1-\left(1-\left(-\beta_{\tau_{1}}^{-}\right)\right)^{\delta}\right),-\left(1-\left(1-\left(-\gamma_{\tau_{1}}^{-}\right)\right)^{\delta}\right)\right\rangle ; \\
\tau_{1}^{\delta}=\left\langle\left(\alpha_{\tau_{1}}^{+}\right)^{\delta}, 1-\left(1-\beta_{\tau_{1}}^{+}\right)^{\delta}, 1-\left(1-\gamma_{\tau_{1}}^{+}\right)^{\delta},-\left(1-\left(1-\left(-\alpha_{\tau_{1}}^{-}\right)\right)^{\delta}\right),-\left(-\beta_{\tau_{1}}^{-}\right)^{\delta},-\left(-\gamma_{\tau_{1}}^{-}\right)^{\delta}\right\rangle .
\end{gathered}
$$

Definition 4 [16]. Let $\tau=\left\langle\alpha_{\tau}^{+}, \beta_{\tau}^{+}, \gamma_{\tau}^{+}, \alpha_{\tau}^{-}, \beta_{\tau}^{-}, \gamma_{\tau}^{-}\right\rangle$be a BNN; then, we define $s(\tau), a(\tau)$, and $c(\tau)$ as the score, accuracy, and certain functions, respectively; they are as follows:

$$
\begin{gathered}
s(\tau)=\frac{1}{6}\left(\alpha_{\tau}^{+}+1-\beta_{\tau}^{+}+1-\gamma_{\tau}^{+}+1+\alpha_{\tau}^{-}-\beta_{\tau}^{-}-\gamma_{\tau}^{-}\right) ; \\
a(\tau)=\alpha_{\tau}^{+}-\gamma_{\tau}^{+}+\alpha_{\tau}^{-}-\gamma_{\tau}^{-} ; \\
c(\tau)=\alpha_{\tau}^{+}-\gamma_{\tau}^{+} .
\end{gathered}
$$

Definition 5 [16]. Let $\tau_{1}$ and $\tau_{2}$ be two BNNs, $\tau_{1}\left\langle=\alpha_{\tau_{1}}^{+}, \beta_{\tau_{1}}^{+}, \gamma_{\tau_{1}}^{+}, \alpha_{\tau_{1}}^{-}, \beta_{\tau_{1}}^{-}, \gamma_{\tau_{1}}^{-}\right\rangle$and $\tau_{2}=$ $\left\langle\alpha_{\tau_{2}}^{+}, \beta_{\tau_{2}}^{+}, \gamma_{\tau_{2}}^{+}, \alpha_{\tau_{2}}^{-}, \beta_{\tau_{2}}^{-}, \gamma_{\tau_{2}}^{-}\right\rangle$; then, we can get Figure 1 .

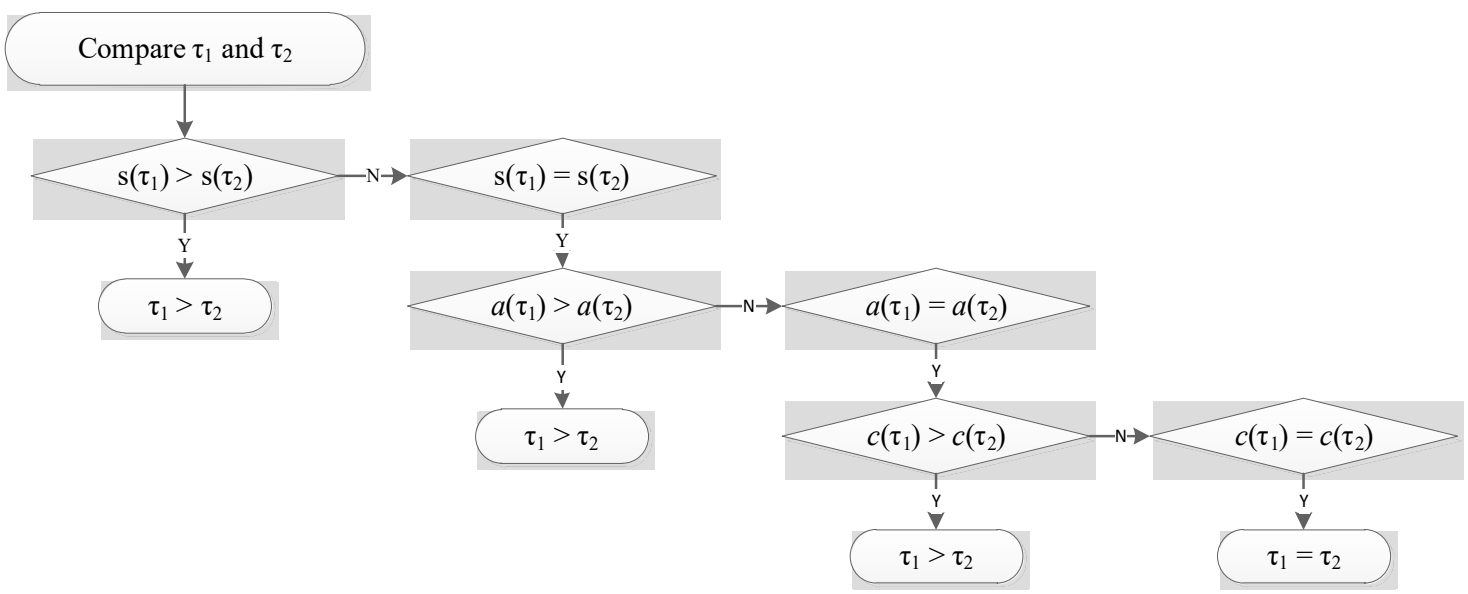

Figure 1. The relationship between $\tau_{1}$ and $\tau_{2}$. 
2.2. Generalized Weighted HM (GWHM), Improved Generalized Weighted HM (IGWHM), Generalized Weighted Geometry HM (GWGHM), and Improved Generalized Weighted Geometry HM (IGWGHM) Operators

Definition 6 [21]. Let $\varepsilon=\left(\varepsilon_{1}, \varepsilon_{2}, \cdots, \varepsilon_{k}\right)$ be the weight vector of a collection of non-negative real numbers $\left(\tau_{1}, \tau_{2}, \ldots, \tau_{k}\right), \sum_{j=1}^{k} \varepsilon_{j}=1$ and $\varepsilon_{j} \in[0,1]$, and $t, s \geq 0$. Then,

$$
\operatorname{GWHM}^{t, s}\left(\tau_{1}, \tau_{2}, \ldots, \tau_{k}\right)=\left(\frac{2}{k(k+1)} \sum_{j=1}^{k} \sum_{i=j}^{k}\left(\varepsilon_{j} \tau_{j}\right)^{t}\left(\varepsilon_{i} \tau_{i}\right)^{s}\right)^{\frac{1}{t+s}}
$$

which is called a GWHM operator.

Definition 7 [22]. Let $\varepsilon=\left(\varepsilon_{1}, \varepsilon_{2}, \cdots, \varepsilon_{k}\right)$ be the weight vector of a collection of non-negative real numbers $\left(\tau_{1}, \tau_{2}, \ldots, \tau_{k}\right), \sum_{j=1}^{k} \varepsilon_{j}=1$ and $\varepsilon_{j} \in[0,1]$, and $t, s \geq 0$. Then,

$$
\operatorname{GWHM}^{t, s}\left(\tau_{1}, \tau_{2}, \ldots, \tau_{k}\right)=\left(\frac{1}{\lambda} \bigoplus_{j=1}^{k} \bigoplus_{i=j}^{k}\left(\varepsilon_{j}{ }^{t} \varepsilon_{i}{ }^{s} \tau_{j}{ }^{t} \otimes \tau_{i}{ }^{s}\right)\right)^{\frac{1}{t+s}},
$$

where $\lambda=\sum_{j=1}^{k} \sum_{i=j}^{k} \varepsilon_{j}{ }^{t} \varepsilon_{i}{ }^{s}$ is called an IGWHM operator.

Definition 8 [21]. Let $\varepsilon=\left(\varepsilon_{1}, \varepsilon_{2}, \cdots, \varepsilon_{k}\right)$ be the weight vector of a collection of non-negative real numbers $\left(\tau_{1}, \tau_{2}, \ldots, \tau_{k}\right), \sum_{j=1}^{k} \varepsilon_{j}=1$ and $\varepsilon_{j} \in[0,1]$, and $t, s \geq 0$. Then,

$$
\operatorname{GWGHM}^{t, s}\left(\tau_{1}, \tau_{2}, \ldots, \tau_{k}\right)=\frac{1}{t+s} \bigotimes_{j=1}^{k} \otimes_{i=j}^{k}\left(\left(t \tau_{j}\right)^{\varepsilon_{j}} \oplus\left(s \tau_{i}\right)^{\varepsilon_{i}}\right)^{\frac{2}{k(k+1)}},
$$

which is called a GWGHM operator.

Definition 9 [22]. Let $\varepsilon=\left(\varepsilon_{1}, \varepsilon_{2}, \cdots, \varepsilon_{k}\right)$ be the weight vector of a collection of non-negative real numbers $\left(\tau_{1}, \tau_{2}, \ldots, \tau_{k}\right), \sum_{j=1}^{k} \varepsilon_{j}=1$ and $\varepsilon_{j} \in[0,1]$, and $t, s \geq 0$. Then,

$$
\operatorname{IGWGHM} M^{t, s}\left(\tau_{1}, \tau_{2}, \ldots, \tau_{k}\right)=\frac{1}{t+s}\left(\bigotimes_{j=1}^{k} \bigotimes_{i=j}^{k}\left(t \tau_{j} \oplus s \tau_{i}\right)^{\frac{2(k+1-j)}{k(k+1)} \frac{\varepsilon_{i}}{\sum_{m=j}^{k} \varepsilon_{m}}}\right),
$$

which is called an IGWGHM operator.

\section{Some BNN Aggregation Operators}

\subsection{GWHM Operators for BNNs}

Definition 10. Let $t, s \geq 0$, and $t+s \neq 0$, a collection $\tau_{j}=\left\langle\alpha_{\tau_{j}}^{+}, \beta_{\tau_{j}}^{+}, \gamma_{\tau_{j}}^{+}, \alpha_{\tau_{j}}^{-}, \beta_{\tau_{j}}^{-}, \gamma_{\tau_{j}}^{-}\right\rangle(j=1,2, \cdots, k)$ of BNN; then, we define the BNNGWHM operator as follows:

$$
\operatorname{BNNGWHM}^{t, s}\left(\tau_{1}, \tau_{2}, \ldots, \tau_{k}\right)=\left(\frac{2}{k(k+1)} \sum_{j=1}^{k} \sum_{i=j}^{k}\left(\varepsilon_{j} \tau_{j}\right)^{t}\left(\varepsilon_{i} \tau_{i}\right)^{s}\right)^{\frac{1}{t+s}},
$$

where $\sum_{j=1}^{k} \varepsilon_{j}=1$ and $\varepsilon_{j} \in[0,1]$.

According to Definitions 3 and 10, the following theorem can be attained: 
Theorem 1. Set a collection $\tau_{j}=\left\langle\alpha_{\tau_{j}}^{+}, \beta_{\tau_{j}}^{+}, \gamma_{\tau_{j}}^{+}, \alpha_{\tau_{j}}^{-}, \beta_{\tau_{j}}^{-}, \gamma_{\tau_{j}}^{-}\right\rangle(j=1,2, \cdots, k)$ of BNNs, using the $B N N G W H M$ operator; then, the aggregation result is still a BNN, which is given by the following form:

$$
\begin{aligned}
& \operatorname{BNNGWHM} M^{t, s}\left(\tau_{1}, \tau_{2}, \ldots, \tau_{k}\right)=\left(\frac{2}{k(k+1)} \sum_{j=1}^{k} \sum_{i=j}^{k}\left(\varepsilon_{j} \tau_{j}\right)^{t}\left(\varepsilon_{i} \tau_{i}\right)^{s}\right)^{\frac{1}{1+s}}=
\end{aligned}
$$

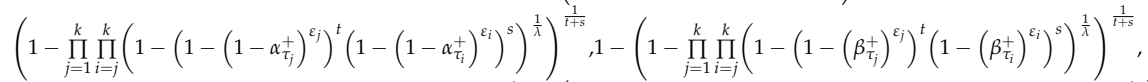

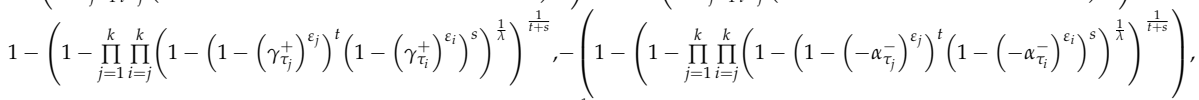

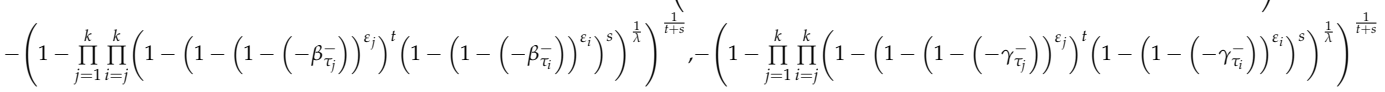

where $\frac{1}{\lambda}=\frac{2}{k(k+1)}, \sum_{j=1}^{k} \varepsilon_{j}=1$ and $\varepsilon_{j} \in[0,1]$.

\section{Proof.}

$$
\begin{aligned}
& \text { (1) } \varepsilon_{j} \tau_{j}=\left\langle 1-\left(1-\alpha_{\tau_{j}}^{+}\right)^{\varepsilon_{j}},\left(\beta_{\tau_{j}}^{+}\right)^{\varepsilon_{j}},\left(\gamma_{\tau_{j}}^{+}\right)^{\varepsilon_{j}},-\left(-\alpha_{\tau_{j}}^{-}\right)^{\varepsilon_{j}},-\left(1-\left(1-\left(-\beta_{\tau_{j}}^{-}\right)\right)^{\varepsilon_{j}}\right),-\left(1-\left(1-\left(-\gamma_{\tau_{j}}^{-}\right)\right)^{\varepsilon_{j}}\right)\right\rangle \text {; } \\
& \text { (2) } \varepsilon_{i} \tau_{i}=\left\langle 1-\left(1-\alpha_{\tau_{i}}^{+}\right)^{\varepsilon_{i}},\left(\beta_{\tau_{i}}^{+}\right)^{\varepsilon_{i}},\left(\gamma_{\tau_{i}}^{+}\right)^{\varepsilon_{i}},-\left(-\alpha_{\tau_{i}}^{-}\right)^{\varepsilon_{i}},-\left(1-\left(1-\left(-\beta_{\tau_{i}}^{-}\right)\right)^{\varepsilon_{i}}\right),-\left(1-\left(1-\left(-\gamma_{\tau_{i}}^{-}\right)\right)^{\varepsilon_{i}}\right)\right\rangle \text {; } \\
& \text { (3) }\left(\varepsilon_{j} \tau_{j}\right)^{t}=\left\langle\left(1-\left(1-\alpha_{\tau_{j}}^{+}\right)^{\varepsilon_{j}}\right)^{t}, 1-\left(1-\left(\beta_{\tau_{j}}^{+}\right)^{\varepsilon_{j}}\right)^{t}, 1-\left(1-\left(\gamma_{\tau_{j}}^{+}\right)^{\varepsilon_{j}}\right)^{t}\right. \text {, } \\
& \left.-\left(1-\left(1-\left(-\alpha_{\tau_{j}}^{-}\right)^{\varepsilon_{j}}\right)^{t}\right),-\left(1-\left(1-\left(-\beta_{\tau_{j}}^{-}\right)\right)^{\varepsilon_{j}}\right)^{t},-\left(1-\left(1-\left(-\gamma_{\tau_{j}}^{-}\right)\right)^{\varepsilon_{j}}\right)^{t}\right\rangle \text {; } \\
& \text { (4) }\left(\varepsilon_{i} \tau_{i}\right)^{s}=\left\langle\left(1-\left(1-\alpha_{\tau_{i}}^{+}\right)^{\varepsilon_{i}}\right)^{s}, 1-\left(1-\left(\beta_{\tau_{i}}^{+}\right)^{\varepsilon_{i}}\right)^{S}, 1-\left(1-\left(\gamma_{\tau_{i}}^{+}\right)^{\varepsilon_{i}}\right)^{s}\right. \text {, } \\
& \left.-\left(1-\left(1-\left(-\alpha_{\tau_{i}}^{-}\right)^{\varepsilon_{i}}\right)^{s}\right),-\left(1-\left(1-\left(-\beta_{\tau_{i}}^{-}\right)\right)^{\varepsilon_{i}}\right)^{s},-\left(1-\left(1-\left(-\gamma_{\tau_{i}}^{-}\right)\right)^{\varepsilon_{i}}\right)^{s}\right\rangle \text {; } \\
& \text { (5) }\left(\varepsilon_{j} \tau_{j}\right)^{t}\left(\varepsilon_{i} \tau_{i}\right)^{s}=\left\langle\left(1-\left(1-\alpha_{\tau_{j}}^{+}\right)^{\varepsilon_{j}}\right)^{t}\left(1-\left(1-\alpha_{\tau_{i}}^{+}\right)^{\varepsilon_{i}}\right)^{s}\right. \text {, } \\
& 1-\left(1-\left(\beta_{\tau_{j}}^{+}\right)^{\varepsilon_{j}}\right)^{t}+1-\left(1-\left(\beta_{\tau_{i}}^{+}\right)^{\varepsilon_{i}}\right)^{s}-\left(1-\left(1-\left(\beta_{\tau_{j}}^{+}\right)^{\varepsilon_{j}}\right)^{t}\right)\left(1-\left(1-\left(\beta_{\tau_{i}}^{+}\right)^{\varepsilon_{i}}\right)^{s}\right), \\
& 1-\left(1-\left(\gamma_{\tau_{j}}^{+}\right)^{\varepsilon_{j}}\right)^{t}+1-\left(1-\left(\gamma_{\tau_{i}}^{+}\right)^{\varepsilon_{i}}\right)^{s}-\left(1-\left(1-\left(\gamma_{\tau_{j}}^{+}\right)^{\varepsilon_{j}}\right)^{t}\right)\left(1-\left(1-\left(\gamma_{\tau_{i}}^{+}\right)^{\varepsilon_{i}}\right)^{s}\right), \\
& -\left(\left(1-\left(1-\left(-\alpha_{\tau_{j}}^{-}\right)^{\varepsilon_{j}}\right)^{t}\right)+\left(1-\left(1-\left(-\alpha_{\tau_{i}}^{-}\right)^{\varepsilon_{i}}\right)^{s}\right)-\left(1-\left(1-\left(-\alpha_{\tau_{j}}^{-}\right)^{\varepsilon_{j}}\right)^{t}\right)\left(1-\left(1-\left(-\alpha_{\tau_{i}}^{-}\right)^{\varepsilon_{i}}\right)^{s}\right)\right) \text {, } \\
& \left.-\left(1-\left(1-\left(-\beta_{\tau_{j}}^{-}\right)\right)^{\varepsilon_{j}}\right)^{t}\left(1-\left(1-\left(-\beta_{\tau_{i}}^{-}\right)\right)^{\varepsilon_{i}}\right)^{s},-\left(1-\left(1-\left(-\gamma_{\tau_{j}}^{-}\right)\right)^{\varepsilon_{j}}\right)^{t}\left(1-\left(1-\left(-\gamma_{\tau_{i}}^{-}\right)\right)^{\varepsilon_{i}}\right)^{s}\right\rangle \\
& \text { (6) } \sum_{j=1}^{k} \sum^{k}{ }_{i=j}\left(\varepsilon_{j} \tau_{j}\right)^{t}\left(\varepsilon_{i} \tau_{i}\right)^{s}=\left\langle 1-\prod_{j=1}^{k} \prod_{i=j}^{k}\left(1-\left(1-\left(1-\alpha_{\tau_{j}}^{+}\right)^{\varepsilon_{j}}\right)^{t}\left(1-\left(1-\alpha_{\tau_{i}}^{+}\right)^{\varepsilon_{i}}\right)^{s}\right)\right. \text {, } \\
& \prod_{j=1}^{k} \prod_{i=j}^{k}\left(1-\left(1-\left(\beta_{\tau_{j}}^{+}\right)^{\varepsilon_{j}}\right)^{t}\left(1-\left(\beta_{\tau_{i}}^{+}\right)^{\varepsilon_{i}}\right)^{S}\right), \prod_{j=1}^{n} \prod_{i=j}^{n}\left(1-\left(1-\left(\gamma_{\tau_{j}}^{+}\right)^{\varepsilon_{j}}\right)^{t}\left(1-\left(\gamma_{\tau_{i}}^{+}\right)^{\varepsilon_{i}}\right)^{S}\right) \text {, } \\
& -\prod_{j=1}^{k} \prod_{i=j}^{k}\left(1-\left(1-\left(-\alpha_{\tau_{j}}^{-}\right)^{\varepsilon_{j}}\right)^{t}\left(1-\left(-\alpha_{\tau_{i}}^{-}\right)^{\varepsilon_{i}}\right)^{s}\right), \\
& -\left(1-\prod_{j=1}^{k} \prod_{i=j}^{k}\left(1-\left(1-\left(1-\left(-\beta_{\tau_{j}}^{-}\right)\right)^{\varepsilon_{j}}\right)^{t}\left(1-\left(1-\left(-\beta_{\tau_{i}}^{-}\right)\right)^{\varepsilon_{i}}\right)^{s}\right)\right) \text {, } \\
& \left.-\left(1-\prod_{j=1}^{k} \prod_{i=j}^{k}\left(1-\left(1-\left(1-\left(-\gamma_{\tau_{j}}^{-}\right)\right)^{\varepsilon_{j}}\right)^{t}\left(1-\left(1-\left(-\gamma_{\tau_{i}}^{-}\right)\right)^{\varepsilon_{i}}\right)^{s}\right)\right)\right\rangle \text {; }
\end{aligned}
$$




$$
\begin{aligned}
& \text { (7) } \frac{2}{k(k+1)} \sum_{j=1}^{k} \sum_{i=j}^{k}\left(\varepsilon_{j} \tau_{j}\right)^{t}\left(\varepsilon_{i} \tau_{i}\right)^{S}=\frac{1}{\lambda} \sum_{j=1}^{k} \sum_{i=j}^{k}\left(\varepsilon_{j} \tau_{j}\right)^{t}\left(\varepsilon_{i} \tau_{i}\right)^{S}= \\
& 1-\prod_{j=1}^{k} \prod_{i=j}^{k}\left(1-\left(1-\left(1-\alpha_{\tau_{j}}^{+}\right)^{\varepsilon_{j}}\right)^{t}\left(1-\left(1-\alpha_{\tau_{i}}^{+}\right)^{\varepsilon_{i}}\right)^{S}\right)^{\frac{1}{\lambda}}, \\
& \prod_{j=1}^{k} \prod_{i=j}^{k}\left(1-\left(1-\left(\beta_{\tau_{j}}^{+}\right)^{\varepsilon_{j}}\right)^{t}\left(1-\left(\beta_{\tau_{i}}^{+}\right)^{\varepsilon_{i}}\right)^{s}\right)^{\frac{1}{\lambda}}, \\
& \prod_{j=1}^{k} \prod_{i=j}^{k}\left(1-\left(1-\left(\gamma_{\tau_{j}}^{+}\right)^{\varepsilon_{j}}\right)^{t}\left(1-\left(\gamma_{\tau_{i}}^{+}\right)^{\varepsilon_{i}}\right)^{s}\right)^{\frac{1}{\lambda}} \text {, } \\
& -\prod_{j=1}^{k} \prod_{i=j}^{k}\left(1-\left(1-\left(-\alpha_{\tau_{j}}^{-}\right)^{\varepsilon_{j}}\right)^{t}\left(1-\left(-\alpha_{\tau_{i}}^{-}\right)^{\varepsilon_{i}}\right)^{s}\right)^{\frac{1}{\lambda}} \text {, } \\
& -\left(1-\prod_{j=1}^{k} \prod_{i=j}^{k}\left(1-\left(1-\left(1-\left(-\beta_{\tau_{j}}^{-}\right)\right)^{\varepsilon_{j}}\right)^{t}\left(1-\left(1-\left(-\beta_{\tau_{i}}^{-}\right)\right)^{\varepsilon_{i}}\right)^{s}\right)^{\frac{1}{\lambda}}\right) \text {, } \\
& -\left(1-\prod_{j=1}^{k} \prod_{i=j}^{k}\left(1-\left(1-\left(1-\left(-\gamma_{\tau_{j}}^{-}\right)\right)^{\varepsilon_{j}}\right)^{t}\left(1-\left(1-\left(-\gamma_{\tau_{i}}^{-}\right)\right)^{\varepsilon_{i}}\right)^{s}\right)^{\frac{1}{\lambda}}\right) \\
& \text { (8) }\left(\frac{1}{\lambda} \sum_{j=1}^{k} \sum_{i=j}^{k}\left(\varepsilon_{j} \tau_{j}\right)^{t}\left(\varepsilon_{i} \tau_{i}\right)^{s}\right)^{\frac{1}{t+s}}= \\
& \left(1-\prod_{j=1}^{k} \prod_{i=j}^{k}\left(1-\left(1-\left(1-\alpha_{\tau_{j}}^{+}\right)^{\varepsilon_{j}}\right)^{t}\left(1-\left(1-\alpha_{\tau_{i}}^{+}\right)^{\varepsilon_{i}}\right)^{s}\right)^{\frac{1}{\lambda}}\right)^{\frac{1}{t+s}}, \\
& 1-\left(1-\prod_{j=1}^{k} \prod_{i=j}^{k}\left(1-\left(1-\left(\beta_{\tau_{j}}^{+}\right)^{\varepsilon_{j}}\right)^{t}\left(1-\left(\beta_{\tau_{i}}^{+}\right)^{\varepsilon_{i}}\right)^{s}\right)^{\frac{1}{\lambda}}\right)^{\frac{1}{t+s}}, \\
& 1-\left(1-\prod_{j=1}^{k} \prod_{i=j}^{k}\left(1-\left(1-\left(\gamma_{\tau_{j}}^{+}\right)^{\varepsilon_{j}}\right)^{t}\left(1-\left(\gamma_{\tau_{i}}^{+}\right)^{\varepsilon_{i}}\right)^{S}\right)^{\frac{1}{\lambda}}\right)^{\frac{1}{t+s}}, \\
& -\left(1-\left(1-\prod_{j=1}^{k} \prod_{i=j}^{k}\left(1-\left(1-\left(-\alpha_{\tau_{j}}^{-}\right)^{\varepsilon_{j}}\right)^{t}\left(1-\left(-\alpha_{\tau_{i}}^{-}\right)^{\varepsilon_{i}}\right)^{\mathcal{S}}\right)^{\frac{1}{\lambda}}\right)^{\frac{1}{t+s}}\right) \text {, } \\
& -\left(1-\prod_{j=1}^{k} \prod_{i=j}^{k}\left(1-\left(1-\left(1-\left(-\beta_{\bar{\tau}_{j}}^{-}\right)\right)^{\varepsilon_{j}}\right)^{t}\left(1-\left(1-\left(-\beta_{\tau_{i}}^{-}\right)\right)^{\varepsilon_{i}}\right)^{s}\right)^{\frac{1}{\lambda}}\right)^{\frac{1}{t+s}} \\
& -\left(1-\prod_{j=1}^{k} \prod_{i=j}^{k}\left(1-\left(1-\left(1-\left(-\gamma_{\tau_{j}}^{-}\right)\right)^{\varepsilon_{j}}\right)^{t}\left(1-\left(1-\left(-\gamma_{\tau_{i}}^{-}\right)\right)^{\varepsilon_{i}}\right)^{S}\right)^{\frac{1}{\lambda}}\right)^{\frac{1}{t+s}}
\end{aligned}
$$

This proves Theorem 1.

Theorem 2. (Monotonicity). Set $\tau_{j}=\left\langle\alpha_{\tau_{j}}^{+}, \beta_{\tau_{j}}^{+}, \gamma_{\tau_{j}}^{+}, \alpha_{\tau_{j}}^{-}, \beta_{\tau_{j}}^{-}, \gamma_{\tau_{j}}^{-}\right\rangle(j=1,2, \cdots, k)$ and $\sigma_{j}=$ $\left\langle\alpha_{\sigma_{j}}^{+} \beta_{\sigma_{j}}^{+} \gamma_{\sigma_{j^{\prime}}}^{+} \alpha_{\sigma_{j}}^{-} \beta_{\sigma_{j^{\prime}}}^{-} \gamma_{\sigma_{j}}^{-}\right\rangle(j=1,2, \cdots, k)$ as two collections of BNNs; if $\alpha_{\tau_{j}}^{+} \leq \alpha_{\sigma_{j^{\prime}}}^{+} \beta_{\tau_{j}}^{+} \geq \beta_{\sigma_{j^{\prime}}}^{+} \gamma_{\tau_{j}}^{+} \geq$ $\gamma_{\sigma_{j}}^{+}$and $\alpha_{\tau_{j}}^{-} \geq \alpha_{\sigma_{j}}^{-}, \beta_{\tau_{j}}^{-} \leq \beta_{\sigma_{j}}^{-} \gamma_{\tau_{j}}^{-} \leq \gamma_{\sigma_{j}}^{-}$, then

$$
\operatorname{BNNGWHM}^{t, s}\left(\tau_{1}, \tau_{2}, \ldots, \tau_{k}\right) \leq \operatorname{BNNGWHM}^{t, s}\left(\sigma_{1}, \sigma_{2}, \ldots, \sigma_{k}\right) .
$$

Proof. For $\alpha_{\tau_{j}}^{+} \leq \alpha_{\sigma_{j^{\prime}}}^{+} \beta_{\tau_{j}}^{+} \geq \beta_{\sigma_{j^{\prime}}}^{+} \gamma_{\tau_{j}}^{+} \geq \gamma_{\sigma_{j}}^{+}$and $\alpha_{\tau_{j}}^{-} \geq \alpha_{\sigma_{j^{\prime}}}^{-} \beta_{\tau_{j}}^{-} \leq \beta_{\sigma_{j^{\prime}}}^{-} \gamma_{\tau_{j}}^{-} \leq \gamma_{\sigma_{j^{\prime}}}^{-}$it is obvious that

$$
\left(1-\left(1-\alpha_{\tau_{j}}^{+}\right)^{\varepsilon_{j}}\right)^{t}\left(1-\left(1-\alpha_{\tau_{i}}^{+}\right)^{\varepsilon_{i}}\right)^{s} \leq\left(1-\left(1-\alpha_{\sigma_{j}}^{+}\right)^{\varepsilon_{j}}\right)^{t}\left(1-\left(1-\alpha_{\sigma_{i}}^{+}\right)^{\varepsilon_{i}}\right)^{s},
$$




$$
\begin{aligned}
& \left(1-\prod_{j=1}^{k} \prod_{i=j}^{k}\left(1-\left(1-\left(1-\alpha_{\tau_{j}}^{+}\right)^{\varepsilon_{j}}\right)^{t}\left(1-\left(1-\alpha_{\tau_{i}}^{+}\right)^{\varepsilon_{i}}\right)^{s}\right)^{\frac{1}{\lambda}}\right)^{\frac{1}{t+s}} \\
\leq & \left(1-\prod_{j=1}^{k} \prod_{i=j}^{k}\left(1-\left(1-\left(1-\alpha_{\sigma_{j}}^{+}\right)^{\varepsilon_{j}}\right)^{t}\left(1-\left(1-\alpha_{\sigma_{i}}^{+}\right)^{\varepsilon_{i}}\right)^{s}\right)^{\frac{1}{\lambda}}\right)^{\frac{1}{t+s}} .
\end{aligned}
$$

Similarly

$$
\begin{gathered}
1-\left(1-\prod_{j=1}^{k} \prod_{i=j}^{k}\left(1-\left(1-\left(\beta_{\tau_{j}}^{+}\right)^{\varepsilon_{j}}\right)^{t}\left(1-\left(\beta_{\tau_{i}}^{+}\right)^{\varepsilon_{i}}\right)^{s}\right)^{\frac{1}{\lambda}}\right)^{\frac{1}{t+s}} \geq \\
1-\left(1-\prod_{j=1}^{k} \prod_{i=j}^{k}\left(1-\left(1-\left(\beta_{\sigma_{j}}^{+}\right)^{\varepsilon_{j}}\right)^{t}\left(1-\left(\beta_{\sigma_{i}}^{+}\right)^{\varepsilon_{i}}\right)^{s}\right)^{\frac{1}{\lambda}}\right)^{\frac{1}{t+s}}, \\
1-\left(1-\prod_{j=1}^{k} \prod_{i=j}^{k}\left(1-\left(1-\left(\gamma_{\tau_{j}}^{+}\right)^{\varepsilon_{j}}\right)^{t}\left(1-\left(\gamma_{\tau_{i}}^{+}\right)^{\varepsilon_{i}}\right)^{s}\right)^{\frac{1}{\lambda}}\right)^{\frac{1}{t+s}} \\
\geq 1-\left(1-\prod_{j=1}^{k} \prod_{i=j}^{k}\left(1-\left(1-\left(\gamma_{\sigma_{j}}^{+}\right)^{\varepsilon_{j}}\right)^{t}\left(1-\left(\gamma_{\sigma_{i}}^{+}\right)^{\varepsilon_{i}}\right)^{s}\right)^{\frac{1}{\lambda}}\right)^{\frac{1}{t+s}}, \\
-\left(1-\left(1-\prod_{j=1}^{k} \prod_{i=j}^{k}\left(1-\left(1-\left(-\alpha_{\tau_{j}}^{-}\right)^{\varepsilon_{j}}\right)^{t}\left(1-\left(-\alpha_{\tau_{i}}^{-}\right)^{\varepsilon_{i}}\right)^{s}\right)^{\frac{1}{\lambda}}\right)^{\frac{1}{t+s}}\right) \\
-\left(1-\left(1-\prod_{j=1}^{k} \prod_{i=j}^{k}\left(1-\left(1-\left(-\alpha_{\sigma_{j}}^{-}\right)^{\varepsilon_{j}}\right)^{t}\left(1-\left(-\alpha_{\sigma_{i}}^{-}\right)^{\varepsilon_{i}}\right)^{s}\right)^{\frac{1}{\lambda}}\right)^{\frac{1}{t+s}}\right) \\
\leq-\left(1-\prod_{j=1}^{k} \prod_{i=j}^{k}\left(1-\left(1-\left(1-\left(-\beta_{\tau_{j}}^{-}\right)\right)^{\varepsilon_{j}}\right)^{t}\left(1-\left(1-\left(-\beta_{\tau_{i}}^{-}\right)\right)^{\varepsilon_{i}}\right)^{s}\right)^{\frac{1}{\lambda}} \prod^{\frac{1}{t+s}}\right. \\
\leq \prod_{i=j}^{k}\left(1-\left(1-\left(1-\left(-\beta_{\sigma_{j}}^{-}\right)\right)^{\varepsilon_{j}}\right)^{t}\left(1-\left(1-\left(-\beta_{\sigma_{i}}^{-}\right)^{\varepsilon_{i}}\right)^{s}\right)^{\frac{1}{\lambda}}\right)^{\frac{1}{t+s}}
\end{gathered}
$$

and

$$
\begin{aligned}
& -\left(1-\prod_{j=1}^{k} \prod_{i=j}^{k}\left(1-\left(1-\left(1-\left(-\gamma_{\tau_{j}}^{-}\right)\right)^{\varepsilon_{j}}\right)^{t}\left(1-\left(1-\left(-\gamma_{\tau_{i}}^{-}\right)\right)^{\varepsilon_{i}}\right)^{s}\right)^{\frac{1}{\lambda}}\right)^{\frac{1}{s+t}} \leq \\
& -\left(1-\prod_{j=1}^{k} \prod_{i=j}^{k}\left(1-\left(1-\left(1-\left(-\gamma_{\sigma_{j}}^{-}\right)\right)^{\varepsilon_{j}}\right)^{t}\left(1-\left(1-\left(-\gamma_{\sigma_{i}}^{-}\right)\right)^{\varepsilon_{i}}\right)^{s}\right)^{\frac{1}{\lambda}}\right)^{\frac{1}{s+t}} .
\end{aligned}
$$

Thus, $B N N G W H M^{t, s}\left(\tau_{1}, \tau_{2}, \ldots, \tau_{k}\right) \leq B N N G W H M^{t, s}\left(\sigma_{1}, \sigma_{2}, \ldots, \sigma_{k}\right)$; this proves Theorem 2 .

\subsection{Improved Generalized Weighted HM Operators for BNNs}

Definition 11. Let $t, s \geq 0$, and $t+s \neq 0$, a collection $\tau_{j}\left\langle=\alpha_{\tau_{j}}^{+}, \beta_{\tau_{j^{\prime}}}^{+}, \gamma_{\tau_{j}}^{+}, \alpha_{\tau_{j}}^{-}, \beta_{\tau_{j^{\prime}}}^{-} \gamma_{\tau_{j}}^{-}(j=1,2, \cdots, k)\right\rangle$ of $B N N$; then, we define the BNNIGWHM operator as follows:

$$
\operatorname{BNNIGWHM} M^{t, s}\left(\tau_{1}, \tau_{2}, \ldots, \tau_{k}\right)=\left(\frac{1}{\sum_{j=1}^{k} \sum_{i=j}^{k} \varepsilon_{j} \varepsilon_{i}} \stackrel{\oplus}{j}=1_{i=j}^{k} \bigoplus_{i=j}^{k}\left(\varepsilon_{j} \varepsilon_{i} \tau_{j}^{t} \otimes \tau_{i}^{s}\right)\right)^{\frac{1}{t+s}},
$$

where $\sum_{j=1}^{k} \varepsilon_{j}=1$ and $\varepsilon_{j} \in[0,1]$. 
According to Definitions 3 and 11, the following theorem can be attained:

Theorem 3. Set a collection $\tau_{j}\left\langle=\alpha_{\tau_{j}}^{+}, \beta_{\tau_{j}}^{+}, \gamma_{\tau_{j}}^{+}, \alpha_{\tau_{j}}^{-}, \beta_{\tau_{j}}^{-}, \gamma_{\tau_{j}}^{-}(j=1,2, \cdots, k)\right\rangle$ of BNNs, using BNNIGWHM operator; then, the aggregation result is still a BNN, which is given by the following form:

$$
\begin{aligned}
& \operatorname{BNNIGWHM}^{t, s}\left(\tau_{1}, \tau_{2}, \ldots, \tau_{k}\right)=\left(\frac{1}{\sum_{j=1}^{k} \sum_{i=j}^{k} \varepsilon_{j} \varepsilon_{i}} \bigoplus_{j=1}^{k} \bigoplus_{i=j}^{k}\left(\varepsilon_{j} \varepsilon_{i} \tau_{j}^{t} \otimes \tau_{i}^{s}\right)\right)^{\frac{1}{t+s}}= \\
& \left(1-\left(\prod_{j=1}^{k} \prod_{i=j}^{k}\left(1-\left(\alpha_{\tau_{j}}^{+}\right)^{t}\left(\alpha_{\tau_{i}}^{+}\right)^{s}\right)^{\varepsilon_{j} \varepsilon_{i}}\right)^{\frac{1}{\lambda}}\right)^{\frac{1}{t+s}} \\
& 1-\left(1-\left(\prod_{j=1}^{k} \prod_{i=j}^{k}\left(1-\left(1-\beta_{\tau_{j}}^{+}\right)^{t}\left(1-\beta_{\tau_{i}}^{+}\right)^{\mathcal{S}}\right)^{\varepsilon_{j} \varepsilon_{i}}\right)^{\frac{1}{\lambda}}\right)^{\frac{1}{t+s}}, \\
& 1-\left(1-\left(\prod_{j=1}^{k} \prod_{i=j}^{k}\left(1-\left(1-\gamma_{\tau_{j}}^{+}\right)^{t}\left(1-\gamma_{\tau_{i}}^{+}\right)^{s}\right)^{\varepsilon_{j} \varepsilon_{i}}\right)^{\frac{1}{\lambda}}\right)^{\frac{1}{t+s}} \\
& -\left(1-\left(1-\left(\prod_{j=1}^{k} \prod_{i=j}^{k}\left(1-\left(1-\left(-\alpha_{\tau_{j}}^{-}\right)\right)^{t}\left(1-\left(-\alpha_{\tau_{i}}^{-}\right)\right)^{s}\right)^{\varepsilon_{j} \varepsilon_{i}}\right)^{\frac{1}{\lambda}}\right)^{\frac{1}{t+s}}\right)^{\rangle} \\
& -\left(1-\left(\prod_{j=1}^{k} \prod_{i=j}^{k}\left(1-\left(-\beta_{\tau_{j}}^{-}\right)^{t}\left(-\beta_{\tau_{i}}^{-}\right)^{s}\right)^{\varepsilon_{j} \varepsilon_{i}}\right)^{\frac{1}{\lambda}}\right)^{\frac{1}{t+s}} \\
& -\left(1-\left(\prod_{j=1}^{k} \prod_{i=j}^{k}\left(1-\left(-\gamma_{\tau_{j}}\right)^{t}\left(-\gamma_{\tau_{i}}\right)^{\mathcal{S}}\right)^{\varepsilon_{j} \varepsilon_{i}}\right)^{\frac{1}{\lambda}}\right)^{\frac{1}{t+s}}
\end{aligned}
$$

where $\lambda=\sum_{j=1}^{k} \sum_{i=j}^{k} \varepsilon_{j} \varepsilon_{i}, \quad \sum_{j=1}^{k} \varepsilon_{j}=1$ and $\varepsilon_{j} \in[0,1]$. it here.

The proof of Theorem 3 can be achieved according to the proof of Theorem 1; thus, we omit

Theorem 4. (Idempotency). Set a collection $\tau_{j}=\left\langle\alpha_{\tau_{j}}^{+}, \beta_{\tau_{j}}^{+}, \gamma_{\tau_{j}}^{+}, \alpha_{\tau_{j}}^{-}, \beta_{\tau_{j}}^{-}, \gamma_{\tau_{j}}^{-}\right\rangle(j=1,2, \cdots, k)$ of BNNs; if $\tau_{j}=\tau$, then

$$
\operatorname{BNNIGWHM}^{t, s}\left(\tau_{1}, \tau_{2}, \ldots, \tau_{k}\right)=\operatorname{BNNIGWHM}^{t, s}(\tau, \tau, \ldots \tau)=\tau \text {. }
$$


Proof. For $\tau_{j}=\tau(j=1,2, \ldots, k)$, the following result can be easily attained:

$$
\begin{aligned}
& \operatorname{BNNIGWHM}^{t, s}\left(\tau_{1}, \tau_{2}, \ldots, \tau_{k}\right)=\operatorname{BNNIGWHM}^{t, s}(\tau, \tau, \ldots \tau)= \\
& =\left(1-\left(\prod_{j=1}^{k} \prod_{i=j}^{k}\left(1-\left(\alpha_{\tau}^{+}\right)^{t}\left(\alpha_{\tau}^{+}\right)^{s}\right)^{\varepsilon_{j} \varepsilon_{i}}\right)^{\frac{1}{\lambda}}\right)^{\frac{1}{t+s}} \\
& 1-\left(1-\left(\prod_{j=1}^{k} \prod_{i=j}^{k}\left(1-\left(1-\beta_{\tau}^{+}\right)^{t}\left(1-\beta_{\tau}^{+}\right)^{s}\right)^{\varepsilon_{j} \varepsilon_{i}}\right)^{\frac{1}{\lambda}}\right)^{\frac{1}{t+s}}, \\
& 1-\left(1-\left(\prod_{j=1}^{k} \prod_{i=j}^{k}\left(1-\left(1-\gamma_{\tau}^{+}\right)^{t}\left(1-\gamma_{\tau}^{+}\right)^{s}\right)^{\varepsilon_{j} \varepsilon_{i}}\right)^{\frac{1}{\lambda}}\right)^{\frac{1}{t+s}}, \\
& -\left(1-\left(1-\left(\prod_{j=1}^{k} \prod_{i=j}^{k}\left(1-\left(1-\left(-\alpha_{\tau}^{-}\right)\right)^{t}\left(1-\left(-\alpha_{\tau}^{-}\right)\right)^{s}\right)^{\varepsilon_{j} \varepsilon_{i}}\right)^{\frac{1}{\lambda}}\right)^{\frac{1}{t+s}}\right), \\
& -\left(1-\left(\prod_{j=1}^{k} \prod_{i=j}^{k}\left(1-\left(-\beta_{\tau}^{-}\right)^{t}\left(-\beta_{\tau}^{-}\right)^{s}\right)^{\varepsilon_{j} \varepsilon_{i}}\right)^{\frac{1}{\lambda}}\right)^{\frac{1}{t+s}}, \\
& -\left(1-\left(\prod_{j=1}^{k} \prod_{i=j}^{k}\left(1-\left(-\gamma_{\tau}^{-}\right)^{t}\left(-\gamma_{\tau}^{-}\right)^{s}\right)^{\varepsilon_{j} \varepsilon_{i}}\right)^{\frac{1}{\lambda}}\right)^{\frac{1}{t+s}} \\
& \left(\left(\alpha_{\tau}^{+}\right)^{t+s}\right)^{\frac{1}{t+s}}, 1-\left(\left(1-\beta_{\tau}^{+}\right)^{t+s}\right)^{\frac{1}{t+s}} \\
& =\left\langle 1-\left(\left(1-\gamma_{\tau}^{+}\right)^{t+s}\right)^{\frac{1}{t+s}},-\left(1-\left(\left(1-\left(-\alpha_{\tau}^{-}\right)\right)^{t+s}\right)^{\frac{1}{t+s}}\right),\right\rangle=\left\langle\alpha_{\tau}^{+}, \beta_{\tau}^{+}, \gamma_{\tau}^{+}, \alpha_{\tau}^{-}, \beta_{\tau}^{-}, \gamma_{\tau}^{-}\right\rangle=\tau \\
& -\left(\left(-\beta_{\tau}^{-}\right)^{t+s}\right)^{\frac{1}{t+s}},-\left(\left(-\gamma_{\tau}^{-}\right)^{t+s}\right)^{\frac{1}{t+s}}
\end{aligned}
$$

This proves Theorem 4 .

Theorem 5. (Monotonicity). Set $\tau_{j}=\left\langle\alpha_{\tau_{j^{\prime}}}^{+}, \beta_{\tau_{\tau^{\prime}}}^{+} \gamma_{\tau_{j}}^{+}, \alpha_{\tau_{j}}^{-} \beta_{\tau_{\tau^{\prime}}}^{-} \gamma_{\tau_{j}}^{-}\right\rangle(j=1,2, \cdots, k)$ and $\sigma_{j}=$ $\left\langle\alpha_{\sigma_{j}}^{+} \beta_{\sigma_{j^{\prime}}}^{+} \gamma_{\sigma_{j^{\prime}}}^{+} \alpha_{\sigma_{j^{\prime}}}^{-} \beta_{\sigma_{j^{\prime}}}^{-} \gamma_{\sigma_{j}}^{-}\right\rangle(j=1,2, \cdots, k)$ as two collections of BNNs; if $\alpha_{\tau_{j}}^{+} \leq \alpha_{\sigma_{\sigma^{\prime}}}^{+} \beta_{\tau_{j}}^{+} \geq \beta_{\sigma_{j^{\prime}}}^{+} \gamma_{\tau_{j}}^{+} \geq$ $\gamma_{\sigma_{j}}^{+}$and $\alpha_{\tau_{j}}^{-} \geq \alpha_{\sigma_{j}}^{-} \beta_{\tau_{j}}^{-} \leq \beta_{\sigma_{j^{\prime}}}^{-} \gamma_{\tau_{j}}^{-} \leq \gamma_{\sigma_{j}}^{-}$, then,

$$
\operatorname{BNNIGWHM}^{t, s}\left(\tau_{1}, \tau_{2}, \ldots, \tau_{k}\right) \leq \text { BNNIGWHM }^{t, s}\left(\sigma_{1}, \sigma_{2}, \ldots, \sigma_{k}\right) .
$$

The proof of Theorem 5 is similar to Theorem 2; thus, we omit it.

Theorem 6. (Boundedness). Set a collection $\tau_{j}=\left\langle\alpha_{\tau_{j}}^{+}, \beta_{\tau_{\tau_{j}}}^{+}, \gamma_{\tau_{j}}^{+}, \alpha_{\tau_{j}}^{-}, \beta_{\tau_{j^{\prime}}}^{-}, \gamma_{\tau_{j}}^{-}\right\rangle(j=1,2, \cdots, k)$ of BNNs, and let $\tau^{-}=\left\langle\begin{array}{l}\min \left(\alpha_{\tau_{j}}^{+}\right), \max \left(\beta_{\tau_{j}}^{+}\right), \max \left(\gamma_{\tau_{j}}^{+}\right), \\ \max \left(\alpha_{\tau_{j}}^{-}\right), \min \left(\beta_{\tau_{j}}^{-}\right), \min \left(\gamma_{\tau_{j}}^{-}\right)\end{array}\right\rangle$and $\tau^{+}=\left\langle\begin{array}{l}\max \left(\alpha_{\tau_{j}}^{+}\right), \min \left(\beta_{\tau_{j}}^{+}\right), \min \left(\gamma_{\tau_{j}}^{+}\right), \\ \min \left(\alpha_{\tau_{j}}^{-}\right), \max \left(\beta_{\tau_{j}}^{-}\right), \max \left(\gamma_{\tau_{j}}^{-}\right)\end{array}\right\rangle ;$then,

$$
\tau^{-} \leq B N N I G W H M^{t, s}\left(\tau_{1}, \tau_{2}, \ldots, \tau_{k}\right) \leq \tau^{+} .
$$

Based on Theorems 4 and 5, the following can be obtained:

$$
\begin{gathered}
\tau^{-}=B N N I G W H M^{t, s}\left(\tau^{-}, \tau^{-}, \ldots, \tau^{-}\right) \text {and } \tau^{+}=\operatorname{BNNIGWHM} M^{t, s}\left(\tau^{+}, \tau^{+}, \ldots, \tau^{+}\right) . \\
B N N I G W H M^{t, s}\left(\tau^{-}, \tau^{-}, \ldots, \tau^{-}\right) \leq B N N I G W H M^{t, s}\left(\tau_{1}, \tau_{2}, \ldots, \tau_{k}\right) \\
\leq B N N I G W H M^{t, s}\left(\tau^{+}, \tau^{+}, \ldots, \tau^{+}\right) .
\end{gathered}
$$


Then, $\tau^{-} \leq B N N I G W H M^{t, s}\left(\tau_{1}, \tau_{2}, \ldots, \tau_{k}\right) \leq \tau^{+}$.

This proves Theorem 6 .

\subsection{GWGHM Operators of BNNs}

Definition 12. Let $t, s \geq 0, t+s \neq 0$, a collection $\tau_{j}=\left\langle\alpha_{\tau_{j}}^{+}, \beta_{\tau_{j}}^{+}, \gamma_{\tau_{j}}^{+}, \alpha_{\tau_{j}}^{-}, \beta_{\tau_{j^{\prime}}}^{-}, \gamma_{\tau_{j}}^{-}\right\rangle(j=1,2, \cdots, k)$ of BNNs; then, we define the BNNGWGHM operator as follows:

$$
\operatorname{BNNGWGHM}^{t, s}\left(\tau_{1}, \tau_{2}, \ldots, \tau_{k}\right)=\frac{1}{t+s} \stackrel{\bigotimes}{j=1}_{i=j}^{k} \stackrel{k}{\otimes}\left(\left(t \tau_{j}\right)^{\varepsilon_{j}} \oplus\left(s \tau_{i}\right)^{\varepsilon_{i}}\right)^{\frac{2}{k(k+1)}},
$$

where $\sum_{j=1}^{k} \varepsilon_{j}=1$ and $\varepsilon_{j} \in[0,1]$.

According to Definitions 3 and 12, the following theorem can be attained:

Theorem 7. Set a collection $\tau_{j}=\left\langle\alpha_{\tau_{j}}^{+} \beta_{\tau_{j^{\prime}}}^{+}, \gamma_{\tau_{j}}^{+}, \alpha_{\tau_{j}}^{-}, \beta_{\tau_{j}}^{-}, \gamma_{\tau_{j}}^{-}\right\rangle(j=1,2, \cdots, k)$ of BNNs, using the $B N N G W G H M$ operator; then, the aggregation result is still a BNN, which is given by the following form:

$$
\begin{aligned}
& \operatorname{BNNGWGHM}^{t, s}\left(\tau_{1}, \tau_{2}, \ldots, \tau_{k}\right)=\frac{1}{t+s} \underset{j=1}{\stackrel{\otimes}{\otimes}} \otimes_{i=j}^{k}\left(\left(t \tau_{j}\right)^{\varepsilon_{j}} \oplus\left(s \tau_{i}\right)^{\varepsilon_{i}}\right)^{\frac{2}{k(k+1)}}= \\
& 1-\left(1-\prod_{j=1}^{k} \prod_{i=j}^{k}\left(1-\left(1-\left(1-\left(1-\left(\alpha_{\tau_{j}}^{+}\right)\right)^{t}\right)^{\varepsilon_{j}}\right)\left(1-\left(1-\left(1-\left(\alpha_{\tau_{i}}^{+}\right)\right)^{s}\right)^{\varepsilon_{i}}\right)\right)^{\frac{1}{\lambda}}\right)^{\frac{1}{t+s}}, \\
& \left(1-\prod_{j=1}^{k} \prod_{i=j}^{k}\left(1-\left(1-\left(1-\left(\beta_{\tau_{j}}^{+}\right)^{t}\right)^{\varepsilon_{j}}\right)\left(1-\left(1-\left(\beta_{\tau_{i}}^{+}\right)^{s}\right)^{\varepsilon_{i}}\right)\right)^{\frac{1}{\lambda}}\right)^{\frac{1}{t+s}}, \\
& \left(1-\prod_{j=1}^{k} \prod_{i=j}^{k}\left(1-\left(1-\left(1-\left(\gamma_{\tau_{j}}^{+}\right)^{t}\right)^{\varepsilon_{j}}\right)\left(1-\left(1-\left(\gamma_{\tau_{i}}^{+}\right)^{s}\right)^{\varepsilon_{i}}\right)\right)^{\frac{1}{\lambda}}\right)^{\frac{1}{t+s}}, \\
& -\left(1-\prod_{j=1}^{k} \prod_{i=j}^{k}\left(1-\left(1-\left(1-\left(-\alpha_{\tau_{j}}^{-}\right)^{t}\right)^{\varepsilon_{j}}\right)\left(1-\left(1-\left(-\alpha_{\tau_{i}}^{-}\right)^{s}\right)^{\varepsilon_{i}}\right)\right)^{\frac{1}{\lambda}}\right)^{\frac{1}{t+s}}, \\
& -\left(1-\left(1-\prod_{j=1}^{k} \prod_{i=j}^{k}\left(1-\left(1-\left(1-\left(1-\left(-\beta_{\tau_{j}}^{-}\right)\right)^{t}\right)^{\varepsilon_{j}}\right)\left(1-\left(1-\left(1-\left(-\beta_{\tau_{i}}^{-}\right)\right)^{s}\right)^{\varepsilon_{i}}\right)\right)^{\frac{1}{\lambda}}\right)^{\frac{1}{t+s}}\right), \\
& -\left(1-\left(1-\prod_{j=1}^{k} \prod_{i=j}^{k}\left(1-\left(1-\left(1-\left(1-\left(-\gamma_{\tau_{j}}\right)\right)^{t}\right)^{\varepsilon_{j}}\right)\left(1-\left(1-\left(1-\left(-\gamma_{\tau_{i}}\right)\right)^{s}\right)^{\varepsilon_{i}}\right)\right)^{\frac{1}{\lambda}}\right)^{\frac{1}{t+s}}\right)
\end{aligned}
$$

where $\frac{1}{\lambda}=\frac{2}{k(k+1)}, \quad \sum_{j=1}^{k} \varepsilon_{j}=1$ and $\varepsilon_{j} \in[0,1]$.

Theorem 8. (Monotonicity). Set $\tau_{j}=\left\langle\alpha_{\tau_{j}}^{+} \beta_{\tau_{j^{\prime}}}^{+}, \gamma_{\tau_{j}}^{+}, \alpha_{\tau_{j}}^{-}, \beta_{\tau_{j}}^{-}, \gamma_{\tau_{j}}^{-}\right\rangle(j=1,2, \cdots, k)$ and $\sigma_{j}=$ $\left\langle\alpha_{\sigma_{j^{\prime}}}^{+} \beta_{\sigma_{j^{\prime}}}^{+} \gamma_{\sigma_{j^{\prime}}}^{+} \alpha_{\sigma_{j^{\prime}}}^{-} \beta_{\sigma_{j^{\prime}}}^{-} \gamma_{\sigma_{j}}^{-}\right\rangle(j=1,2, \cdots, k)$ as two collections of BNNs; if $\alpha_{\tau_{j}}^{+} \leq \alpha_{\sigma_{j^{\prime}}}^{+} \beta_{\tau_{j}}^{+} \geq \beta_{\sigma_{j^{\prime}}}^{+}, \gamma_{\tau_{j}}^{+} \geq$ $\gamma_{\sigma_{j}}^{+}$and $\alpha_{\tau_{j}}^{-} \geq \alpha_{\sigma_{j}}^{-} \beta_{\tau_{j}}^{-} \leq \beta_{\sigma_{j^{\prime}}}^{-} \gamma_{\tau_{j}}^{-} \leq \gamma_{\sigma_{j}}^{-}$, then,

$$
\operatorname{BNNGWGHM} M^{t, s}\left(\tau_{1}, \tau_{2}, \ldots, \tau_{k}\right) \leq \operatorname{BNNGWGHM}^{t, s}\left(\sigma_{1}, \sigma_{2}, \ldots, \sigma_{k}\right) .
$$

The proofs of theorems about BNNGWGHM are similar to those about BNNGWHM; thus, we omit them. 


\subsection{IGWGHM Operators of BNNs}

Definition 13. Let $t, s \geq 0$, and $t+s \neq 0$, a collection $\tau_{j}=\left\langle\alpha_{\tau_{j^{\prime}}}^{+}, \beta_{\tau_{j^{\prime}}}^{+} \gamma_{\tau_{j^{\prime}}}^{+}, \alpha_{\tau_{j^{\prime}}}^{-}, \beta_{\tau_{j^{\prime}}}^{-}, \gamma_{\tau_{j}}^{-}\right\rangle(j=1,2, \cdots, k)$ of $B N N$; then, we define the BNNIGWGHM operator as follows:

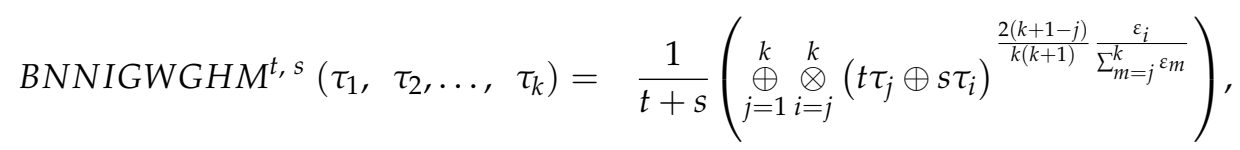

where $\sum_{j=1}^{k} \varepsilon_{j}=1$ and $\varepsilon_{j} \in[0,1]$.

According to Definitions 3 and 13, the following theorem can be attained:

Theorem 9. Set a collection $\tau_{j}=\left\langle\alpha_{\tau_{j}}^{+}, \beta_{\tau_{j^{\prime}}}^{+} \gamma_{\tau_{j^{\prime}}}^{+} \alpha_{\tau_{j^{\prime}}}^{-} \beta_{\tau_{j^{\prime}}}^{-} \gamma_{\tau_{j}}^{-}\right\rangle(j=1,2, \cdots, k)$ of BNNs, using the BNNIGWGHM operator; then, the aggregation result is still a BNN, which is given by the following form:

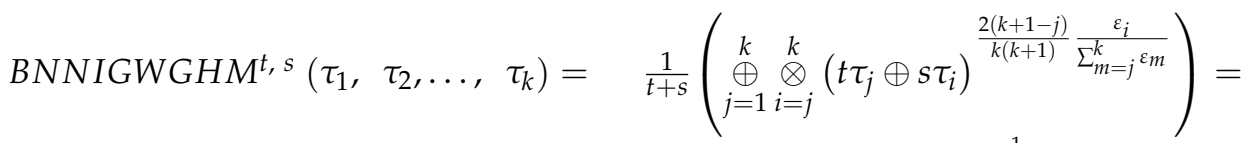

$$
\begin{aligned}
& 1-\left(1-\prod_{j=1}^{k} \prod_{i=j}^{k}\left(1-\left(1-\left(\alpha_{\tau_{j}}^{+}\right)\right)^{t}\left(1-\left(\alpha_{\tau_{i}}^{+}\right)\right)^{s}\right)^{\frac{1}{\lambda}}\right)^{\frac{1}{t+s}}, \\
& \left(1-\prod_{j=1}^{k} \prod_{i=j}^{k}\left(1-\left(\beta_{\tau_{j}}^{+}\right)^{t}\left(\beta_{\tau_{i}}^{+}\right)^{s}\right)^{\frac{1}{\lambda}}\right)^{\frac{1}{t+s}} \\
& \left(1-\prod_{j=1}^{k} \prod_{i=j}^{k}\left(1-\left(\gamma_{\tau_{j}}^{+}\right)^{t}\left(\gamma_{\tau_{i}}^{+}\right)^{s}\right)^{\frac{1}{\lambda}}\right)^{\frac{1}{t+s}} \\
& \left.-\left(1-\prod_{j=1}^{k} \prod_{i=j}^{k}\left(1-\left(-\alpha_{\tau_{j}}^{-}\right)^{t}\left(-\alpha_{\tau_{i}}^{-}\right)^{s}\right)^{\frac{1}{\lambda}}\right)^{\frac{1}{t+s}}, \quad\right\rangle, \\
& -\left(1-\left(1-\prod_{j=1}^{k} \prod_{i=j}^{k}\left(1-\left(1-\left(-\beta_{\tau_{j}}^{-}\right)\right)^{t}\left(1-\left(-\beta_{\tau_{i}}^{-}\right)\right)^{s}\right)^{\frac{1}{\lambda}}\right)^{\frac{1}{t+s}}\right) \text {, } \\
& -\left(1-\left(1-\prod_{j=1}^{k} \prod_{i=j}^{k}\left(1-\left(1-\left(-\gamma_{\tau_{j}}^{-}\right)\right)^{t}\left(1-\left(-\gamma_{\tau_{i}}^{-}\right)\right)^{s}\right)^{\frac{1}{\lambda}}\right)^{\frac{1}{t+s}}\right)
\end{aligned}
$$

where $\frac{1}{\lambda}=\frac{2(k+1-j)}{k(k+1)} \frac{\varepsilon_{i}}{\sum_{m=j}^{k} \varepsilon_{m}}, \quad \sum_{j=1}^{k} \varepsilon_{j}=1$ and $\varepsilon_{j} \in[0,1]$

Theorem 10. (Monotonicity). Set $\tau_{j}=\left\langle\alpha_{\tau_{j^{\prime}}}^{+}, \beta_{\tau_{j^{\prime}}}^{+}, \gamma_{\tau_{j}}^{+}, \alpha_{\tau_{j^{\prime}}}^{-}, \beta_{\tau_{j^{\prime}}}^{-} \gamma_{\tau_{j}}^{-}\right\rangle(j=1,2, \cdots, k)$ and $\sigma_{j}=$ $\alpha_{\sigma_{j^{\prime}}}^{+} \beta_{\sigma_{j^{\prime}}}^{+} \gamma_{\sigma_{j^{\prime}}}^{+} \alpha_{\sigma_{j^{\prime}}}^{-} \beta_{\sigma_{j^{\prime}}}^{-} \gamma_{\sigma_{j}}^{-}(j=1,2, \cdots, k)$ as two collections of BNNs; if $\alpha_{\tau_{j}}^{+} \leq \alpha_{\sigma_{j^{\prime}}}^{+} \beta_{\tau_{j}}^{+} \geq \beta_{\sigma_{j^{\prime}}}^{+} \gamma_{\tau_{j}}^{+} \geq$ $\gamma_{\sigma_{j}}^{+}$and $\alpha_{\tau_{j}}^{-} \geq \alpha_{\sigma_{j}}^{-}, \beta_{\tau_{j}}^{-} \leq \beta_{\sigma_{j}}^{-} \gamma_{\tau_{j}}^{-} \leq \gamma_{\sigma_{j^{\prime}}}^{-}$then,

$$
\operatorname{BNNIGWGHM^{t,s}}\left(\tau_{1}, \tau_{2}, \ldots, \tau_{k}\right) \leq B N N I G W G H M^{t, s}\left(\sigma_{1}, \sigma_{2}, \ldots, \sigma_{k}\right) .
$$

Theorem 11. (Idempotency). Set a collection $\tau_{j}=\left\langle\alpha_{\tau_{j}}^{+}, \beta_{\tau_{j}}^{+}, \gamma_{\tau_{j}}^{+}, \alpha_{\tau_{j^{\prime}}}^{-}, \beta_{\tau_{j}}^{-}, \gamma_{\tau_{j}}^{-}\right\rangle(j=1,2, \cdots, k)$ of BNNs; if $\tau_{j}=\tau$, then,

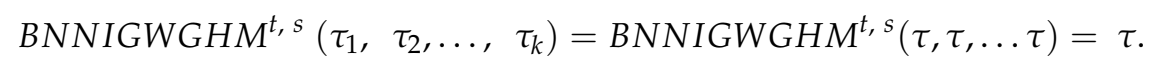


Theorem 12. (Boundedness). Set a collection $\tau_{j}=\left\langle\alpha_{\tau_{j}}^{+}, \beta_{\tau_{j}}^{+}, \gamma_{\tau_{j}}^{+}, \alpha_{\tau_{j}}^{-}, \beta_{\tau_{j}}^{-}, \gamma_{\tau_{j}}^{-}\right\rangle(j=1,2, \cdots, k)$ of $B N N$, and let $\tau^{-}=\left\langle\min \left(\alpha_{\tau_{j}}^{+}\right), \max \left(\beta_{\tau_{j}}^{+}\right), \max \left(\gamma_{\tau_{j}}^{+}\right), \max \left(\alpha_{\tau_{j}}^{-}\right), \min \left(\beta_{\tau_{j}}^{-}\right), \min \left(\gamma_{\tau_{j}}^{-}\right)\right\rangle$, and $\tau^{+}=$ $\left\langle\max \left(\alpha_{\tau_{j}}^{+}\right), \min \left(\beta_{\tau_{j}}^{+}\right), \min \left(\gamma_{\tau_{j}}^{+}\right), \min \left(\alpha_{\tau_{j}}^{-}\right), \max \left(\beta_{\tau_{j}}^{-}\right), \max \left(\gamma_{\tau_{j}}^{-}\right)\right\rangle ;$then,

$$
\tau^{-} \leq B N N I G W H M^{t, s}\left(\tau_{1}, \tau_{2}, \ldots, \tau_{k}\right) \leq \tau^{+}
$$

The proofs of theorems about BNNIGWGHM are similar to those about BNNIGWHM; thus, we omit them.

\section{MCDM Methods Based on the BNNIGWHM and BNNIGWGHM Operator}

We applied the BNNIGWHM and BNNIGWGHM operator to manage MCDM problems within $\mathrm{BNN}$ information in this section.

Suppose that a set $\Gamma=\left\{\Gamma_{1}, \Gamma_{2}, \ldots, \Gamma_{n}\right\}$ of alternatives and a set $\Phi=\left\{\Phi_{1}, \Phi_{2}, \ldots, \Phi_{m}\right\}$ of attributes, with the weight vector $\varepsilon=\left(\varepsilon_{1}, \varepsilon_{2}, \ldots, \varepsilon_{m}\right)$ of $\Phi_{j}(j=1,2, \ldots, m)$, in which $\sum_{j=1}^{n} \varepsilon_{j}=1$ and $\varepsilon_{j} \in[0,1]$. Decision-makers use BNNs to evaluate the alternatives. The evaluation values $\tau_{i j}$ for $\Gamma_{i}$ associated with the attribute $\Phi_{j}$ are represented by the form of BNNs. Assume that $\left(\tau_{i j}\right)_{n \times m}=\left(\left\langle\alpha_{\tau_{i j}}^{+}, \beta_{\tau_{i j}}^{+}, \gamma_{\tau_{i j}}^{+}, \alpha_{\tau_{i j}}^{-}, \beta_{\tau_{i j}}^{-}, \gamma_{\tau_{i j}}^{-}\right\rangle\right)_{n \times m}$ is the BNN decision matrix.

Now, based on the BNNIGWHM and BNNIGWGHM operator, we can develop some decision algorithms:

Step 1: Construct the decision matrix:

$$
\left(\tau_{i j}\right)_{n \times m}=\left(\left\langle\alpha_{\tau_{i j}}^{+}, \beta_{\tau_{i j}}^{+}, \gamma_{\tau_{i j}}^{+}, \alpha_{\tau_{i j}}^{-}, \beta_{\tau_{i j}}^{-}, \gamma_{\tau_{i j}}^{-}\right\rangle\right)_{n \times m}
$$

Step 2: According to Definition 11 or Definition 13, calculate $\tau_{i}$.

Step 3: According to the Equation (5), calculate the score value of $s\left(\tau_{i}\right)$ for $\tau_{i}(i=1,2, \ldots, n)$.

Step 4: According to Definition 5, rank all the alternatives corresponding to the values of $s\left(\tau_{i}\right)$.

\section{Illustrative Example}

In this section, we used a numerical example adapted from the literature [16]. A woman wants to buy a car. Now, four kinds of cars $\Gamma_{1}, \Gamma_{2}, \Gamma_{3}$, and $\Gamma_{4}$ are taken into account according to gasoline consumption $\left(\Phi_{1}\right)$, aerodynamics $\left(\Phi_{2}\right)$, comfort $\left(\Phi_{3}\right)$, and safety performances $\left(\Phi_{4}\right)$. The importance of these four attributes is given as $\varepsilon=(0.5,0.25,0.125,0.125)^{T}$. Then, she evaluates four alternatives under the above four attributes in the form of BNNs.

\subsection{The Decision-Making Process Based on the BNNIGWHM Operator or BNNIGWGHM Operator}

Step 1: Establish the BNN decision matrix $\left(\tau_{i j}\right)_{4 \times 4}$ provided by customer, as shown in Table 1.

Table 1. The decision matrix $\left(\tau_{i j}\right)_{4 \times 4}$.

\begin{tabular}{ccccc}
\hline & $\boldsymbol{\Phi}_{\mathbf{1}}$ & $\boldsymbol{\Phi}_{\mathbf{2}}$ & $\boldsymbol{\Phi}_{\mathbf{3}}$ & $\boldsymbol{\Phi}_{\mathbf{4}}$ \\
\hline$\Gamma_{1}$ & $\langle 0.5,0.7,0.2,-0.7,-0.3,-0.6\rangle$ & $\langle 0.4,0.4,0.5,-0.7,-0.8,-0.4\rangle$ & $\langle 0.7,0.7 .0 .5,-0.8,-0.7,-0.6\rangle$ & $\langle 0.1,0.5,0.7,-0.5,-0.2,-0.8\rangle$ \\
$\Gamma_{2}$ & $\langle 0.9,0.7,0.5,-0.7,-0.7,-0.1\rangle$ & $\langle 0.7,0.6,0.8,-0.7,-0.5,-0.1\rangle$ & $\langle 0.9,0.4,0.6,-0.1,-0.7,-0.5\rangle$ & $\langle 0.5,0.2,0.7,-0.5,-0.1,-0.9\rangle$ \\
$\Gamma_{3}$ & $\langle 0.3,0.4,0.2,-0.6,-0.3,-0.7\rangle$ & $\langle 0.2,0.2,0.2,-0.4,-0.7,-0.4\rangle$ & $\langle 0.9,0.5,0.5,-0.6,-0.5,-0.2\rangle$ & $\langle 0.7,0.5,0.3,-0.4,-0.2,-0.2\rangle$ \\
$\Gamma_{4}$ & $\langle 0.9,0.7,0.2,-0.8,-0.6,-0.1\rangle$ & $\langle 0.3,0.5,0.2,-0.5,-0.5,-0.2\rangle$ & $\langle 0.5,0.4,0.5,-0.1,-0.7,-0.2\rangle$ & $\langle 0.4,0.2,0.8,-0.5,-0.5,-0.6\rangle$ \\
\hline
\end{tabular}

Step 2: According to Definition 11 (suppose $p=q=1$ ) and $\varepsilon$ of attributes, calculate $\tau_{i}(i=1,2,3,4)$ :

$$
\begin{aligned}
& \tau_{1}=\langle 0.4656,0.5984,0.3248,-0.6874,-0.4906,-0.5832\rangle, \\
& \tau_{2}=\langle 0.8362,0.5751,0.5918,-0.5868,-0.6108,-0.2872\rangle,
\end{aligned}
$$




$$
\begin{gathered}
\tau_{3}=\langle 0.4212,0.3684,0.2341,-0.5268,-0.4254,-0.5540\rangle, \\
\tau_{4}=\langle 0.7456,0.5504,0.2669,-0.5838,-0.5793,-0.2006\rangle .
\end{gathered}
$$

Step 3: According to Equation (5), calculate thscore value of $s\left(\tau_{i}\right)$ for $\tau_{i}(i=1,2,3,4)$ :

$$
s\left(\tau_{1}\right)=0.4881 ; s\left(\tau_{2}\right)=0.4968 ; s\left(\tau_{3}\right)=0.5458 ; s\left(\tau_{4}\right)=0.5207
$$

Step 4: According to Definition 5, rank $\Gamma_{3} \succ \Gamma_{4} \succ \Gamma_{2} \succ \Gamma_{1}$ corresponding to $s\left(\tau_{i}\right)$; thus, $\Gamma_{3}$ is the best choice among all the alternatives.

Now, we use the BNNIGWGHM operator ( $\operatorname{set} p=1, q=1$ ) to deal with this problem.

Step 1': Just as described in step 1.

Step 2': According to Definition 13 (suppose $p=q=1$ ) and $\varepsilon$ of attributes, calculate $\tau_{i}(i=1,2,3,4)$ :

$$
\begin{aligned}
& \tau_{1}=\langle 0.3834,0.5909,0.4846,-0.6881,-0.4467,-0.5722\rangle, \\
& \tau_{2}=\langle 0.7371,0.5369,0.6627,-0.5747,-0.4484,-0.2381\rangle, \\
& \tau_{3}=\langle 0.4112,0.3994,0.2991,-0.5106,-0.3982,-0.3551\rangle, \\
& \tau_{4}=\langle 0.4922,0.5086,0.4579,-0.5674,-0.5684,-0.2139\rangle .
\end{aligned}
$$

Step 3': According to Equation (5), calculate the score value of $s\left(\tau_{i}\right)$. for $\tau_{i}(i=1,2,3,4)$ :

$$
s\left(\tau_{1}\right)=0.4398 ; s\left(\tau_{2}\right)=0.4416 ; s\left(\tau_{3}\right)=0.4926 ; s\left(\tau_{4}\right)=0.4568
$$

Step 4': According to Definition 5, rank $\Gamma_{3} \succ \Gamma_{4} \succ \Gamma_{2} \succ \Gamma_{1}$ corresponding to $s\left(\tau_{i}\right)$; thus, $\Gamma_{3}$ is the best choice among all the alternatives.

\subsection{Analyzing the Effects of the Parameters $p$ and $q$}

In this section, we took different parameters $p$ and $q$ for calculating $\tau_{i}(i=1,2,3,4)$ for the alternative $\Gamma_{i}$, and then we analyzed the influence of the parameters $p$ and $q$ for the ranking result. Tables 2 and 3 show the values of $s\left(\tau_{1}\right)$ to $s\left(\tau_{4}\right)$ and the ranking results.

Table 2. Ranking results with different values of $p$ and $q$ based on bipolar neutrosophic number improved generalized weighted Heronian mean (BNNIGWHM) operator.

\begin{tabular}{cccc}
\hline No. & $p, q$ & BNNIGWHM & Ranking \\
\hline 1 & $p=1, q=0$ & $s\left(\tau_{1}\right)=0.4915, s\left(\tau_{2}\right)=0.4782, s\left(\tau_{3}\right)=0.5471, s\left(\tau_{4}\right)=0.5116$ & $\Gamma_{3} \succ \Gamma_{4} \succ \Gamma_{1} \succ \Gamma_{2}$ \\
2 & $p=1, q=0.5$ & $s\left(\tau_{1}\right)=0.4823, s\left(\tau_{2}\right)=0.4809, s\left(\tau_{3}\right)=0.5392, s\left(\tau_{4}\right)=0.5083$ & $\Gamma_{3} \succ \Gamma_{4} \succ \Gamma_{1} \succ \Gamma_{2}$ \\
3 & $p=1, q=2$ & $s\left(\tau_{1}\right)=0.5059, s\left(\tau_{2}\right)=0.5316, s\left(\tau_{3}\right)=0.5658, s\left(\tau_{4}\right)=0.5495$ & $\Gamma_{3} \succ \Gamma_{4} \succ \Gamma_{2} \succ \Gamma_{1}$ \\
4 & $p=0, q=1$ & $s\left(\tau_{1}\right)=0.5021, s\left(\tau_{2}\right)=0.5433, s\left(\tau_{3}\right)=0.5659, s\left(\tau_{4}\right)=0.5517$ & $\Gamma_{3} \succ \Gamma_{4} \succ \Gamma_{2} \succ \Gamma_{1}$ \\
5 & $p=0.5, q=1$ & $s\left(\tau_{1}\right)=0.4871, s\left(\tau_{2}\right)=0.4966, s\left(\tau_{3}\right)=0.5445, s\left(\tau_{4}\right)=0.5215$ & $\Gamma_{3} \succ \Gamma_{4} \succ \Gamma_{2} \succ \Gamma_{1}$ \\
6 & $p=2, q=1$ & $s\left(\tau_{1}\right)=0.4981, s\left(\tau_{2}\right)=0.5161, s\left(\tau_{3}\right)=0.5589, s\left(\tau_{4}\right)=0.5346$ & $\Gamma_{3} \succ \Gamma_{4} \succ \Gamma_{2} \succ \Gamma_{1}$ \\
7 & $p=2, q=2$ & $s\left(\tau_{1}\right)=0.5105, s\left(\tau_{2}\right)=0.5425, s\left(\tau_{3}\right)=0.5730, s\left(\tau_{4}\right)=0.5567$ & $\Gamma_{3} \succ \Gamma_{4} \succ \Gamma_{2} \succ \Gamma_{1}$ \\
\hline
\end{tabular}

Table 3. The ranking with different $p$ and $q$ based on BNN improved generalized weighted geometry HM (BNNIGWGHM) operator.

\begin{tabular}{cccc}
\hline No. & $p, q$ & BNNIGWGHM & Ranking \\
\hline 1 & $p=1, q=0$ & $s\left(\tau_{1}\right)=0.5228, s\left(\tau_{2}\right)=0.5768, s\left(\tau_{3}\right)=0.5967, s\left(\tau_{4}\right)=0.5955$ & $\Gamma_{3} \succ \Gamma_{4} \succ \Gamma_{2} \succ \Gamma_{1}$ \\
2 & $p=1, q=0.5$ & $s\left(\tau_{1}\right)=0.4831, s\left(\tau_{2}\right)=0.4893, s\left(\tau_{3}\right)=0.5358, s\left(\tau_{4}\right)=0.5039$ & $\Gamma_{3} \succ \Gamma_{4} \succ \Gamma_{2} \succ \Gamma_{1}$ \\
3 & $p=1, q=2$ & $s\left(\tau_{1}\right)=0.3834, s\left(\tau_{2}\right)=0.3990, s\left(\tau_{3}\right)=0.4504, s\left(\tau_{4}\right)=0.4160$ & $\Gamma_{3} \succ \Gamma_{4} \succ \Gamma_{2} \succ \Gamma_{1}$ \\
4 & $p=0, q=1$ & $s\left(\tau_{1}\right)=0.4190, s\left(\tau_{2}\right)=0.4376, s\left(\tau_{3}\right)=0.4841, s\left(\tau_{4}\right)=0.4584$ & $\Gamma_{3} \succ \Gamma_{4} \succ \Gamma_{2} \succ \Gamma_{1}$ \\
5 & $p=0.5, q=1$ & $s\left(\tau_{1}\right)=0.4411, s\left(\tau_{2}\right)=0.4492, s\left(\tau_{3}\right)=0.4957, s\left(\tau_{4}\right)=0.4664$ & $\Gamma_{3} \succ \Gamma_{4} \succ \Gamma_{2} \succ \Gamma_{1}$ \\
6 & $p=2, q=1$ & $s\left(\tau_{1}\right)=0.4275, s\left(\tau_{2}\right)=0.4211, s\left(\tau_{3}\right)=0.4791, s\left(\tau_{4}\right)=0.4341$ & $\Gamma_{3} \succ \Gamma_{4} \succ \Gamma_{1} \succ \Gamma_{2}$ \\
7 & $p=2, q=2$ & $s\left(\tau_{1}\right)=0.3873, s\left(\tau_{2}\right)=0.3913, s\left(\tau_{3}\right)=0.4496, s\left(\tau_{4}\right)=0.4057$ & $\Gamma_{3} \succ \Gamma_{4} \succ \Gamma_{2} \succ \Gamma_{1}$ \\
\hline
\end{tabular}


From the decision results based on BNNIGWHM in Table 2, we can see that all the ranking orders are $\Gamma_{3} \succ \Gamma_{4} \succ \Gamma_{1} \succ \Gamma_{2}$ in No. 1-2 and all the ranking orders are $\Gamma_{3} \succ \Gamma_{4} \succ \Gamma_{2} \succ \Gamma_{1}$ in No. 3-7; thus, the best choice is $\Gamma_{3}$. From the decision results based on BNNIGWGHM in Table 3, we can see that the ranking order is $\Gamma_{3} \succ \Gamma_{4} \succ \Gamma_{1} \succ \Gamma_{2}$ in No. 6 and the others are $\Gamma_{3} \succ \Gamma_{4} \succ \Gamma_{2} \succ \Gamma_{1}$; thus, the best choice is also $\Gamma_{3}$.

IGWHM and IGWGHM aggregation operators can take into account the correlation between attribute values and can better reflect the preferences of decision-makers and make the decision results more reasonable and reliable. A BNS has two fully independent parts, one part has three independent positive membership functions and the other has three independent negative membership functions, which can deal with uncertain information containing incompatible polarity. Here, we used the BNNIGWHM and BNNIGWGHM operators to solve real problems and analyze the influences of parameters $p$ and $q$ on the results of decisions, using different parameter values for sorting and comparing the corresponding results. Then, it could be found that the influences of parameters $p$ and $q$ on the results of decisions were small in these both methods. Comparing the results of the two methods, it can be found that their results were consistent; therefore, the proposed methods in this paper have feasibility and generality.

\subsection{Comparison with Related Methods}

In this section, we compared the methods proposed in this paper with other related methods proposed in the literature $[16,19]$. Table 4 lists the ranking results.

Table 4. Decision results based on four aggregation operators.

\begin{tabular}{ccc}
\hline Aggregation Operator & Score Value & Ranking \\
\hline $\begin{array}{c}\text { The bipolar neutrosophic weighted average operator } \\
\text { (Aw) and bipolar neutrosophic weighted geometric } \\
\text { operator (Gw) proposed in Reference [16] }\end{array}$ & $\begin{array}{c}\sigma\left(\tau_{1}\right)=0.50, \sigma\left(\tau_{2}\right)=0.52, \\
\sigma\left(\tau_{3}\right)=0.56, \sigma\left(\tau_{4}\right)=0.54\end{array}$ & $\Gamma_{3} \succ \Gamma_{4} \succ \Gamma_{2} \succ \Gamma_{1}$ \\
\hline $\begin{array}{c}\text { The Similarity measures of bipolar neutrosophic sets } \\
\text { proposed in Reference [19] with the following variables: }\end{array}$ & & \\
\hline$\lambda=0.25$ & $\begin{array}{c}\sigma\left(\tau_{1}\right)=0.24683, \sigma\left(\tau_{2}\right)=0.11778, \\
\sigma\left(\tau_{3}\right)=0.27833, \sigma\left(\tau_{4}\right)=0.21136\end{array} \quad \Gamma_{3} \succ \Gamma_{1} \succ \Gamma_{4} \succ \Gamma_{2}$ \\
\hline$\lambda=0.3$ & $\begin{array}{l}\sigma\left(\tau_{1}\right)=0.27063, \sigma\left(\tau_{2}\right)=0.19497, \\
\sigma\left(\tau_{3}\right)=0.30222, \sigma\left(\tau_{4}\right)=0.22904\end{array} \quad \Gamma_{3} \succ \Gamma_{1} \succ \Gamma_{4} \succ \Gamma_{2}$ \\
\hline$\lambda=0.6$ & $\begin{array}{c}\sigma\left(\tau_{1}\right)=0.41342, \sigma\left(\tau_{2}\right)=0.29803, \\
\sigma\left(\tau_{3}\right)=0.44555, \sigma\left(\tau_{4}\right)=0.33510\end{array} \quad \Gamma_{3} \succ \Gamma_{1} \succ \Gamma_{4} \succ \Gamma_{2}$ \\
\hline$\lambda=0.9$ & $\begin{array}{l}\sigma\left(\tau_{1}\right)=0.55620, \sigma\left(\tau_{2}\right)=0.40109, \\
\sigma\left(\tau_{3}\right)=0.54313, \sigma\left(\tau_{4}\right)=0.44116\end{array} \quad \Gamma_{1} \succ \Gamma_{3} \succ \Gamma_{4} \succ \Gamma_{2}$ \\
\hline
\end{tabular}

In Table 4, we can see that the ranking results were different; $\Gamma_{3}$ was obtained as the optimal alternative except the method in Reference [19] with $\lambda=0.9$. Compared with these related methods, the BNNIGWHM and BNNIGWGHM operators considered the correlation between attribute values and could better reflect the preferences of decision-makers and make the decision results more reasonable and reliable while dealing with uncertain information containing incompatible polarity. Thus, we think the proposed methods in this paper are more suitable to handle these decision-making problems.

\section{Conclusions}

This paper firstly proposed the BNNGWHM, BNNIGWHM, BNNGWGHM, and BNNIGWGHM operators for BNNs and discussed the related properties of these four operators. Furthermore, we developed two methods of MCDM in a BNN environment based on the BNNIGWHM and BNNIGWGHM operators. Finally, these two methods were used for a numerical example to establish their effectiveness and application. Dealing with the calculation, we took different values for $p$ and 
$q$ to observe the sorting results and found that both parameters had little influence on the decision results. Furthermore, we compared the proposed methods with related methods and discovered that the selection result using the proposed methods was the same as the majority of existing methods. In the future, we will make further research bipolar neutrosophic sets, using, e.g., the technique for order preference by similarity to an ideal solution (TOPSIS) and VIKOR (VIseKriterijumska Optimizacija I Kompromisno Resenje, that means: multicriteria optimization and compromise solution, with pronunciation: vikor) methods with BNS [23], the weighted aggregated sum product assessment (WASPAS) method with BNS [24], the Multi-Attribute Market Value Assessment ( MAMVA) method with BNS [25], and so on [26-28].

Author Contributions: C.F. proposed the BNNIGWHM and BNNIGWGHM operators and investigated their properties, C.F., S.F. and K.H. presented the organization and decision making method of this paper, J.Y. and E.F. provided the calculation and analysis of the illustrative example; All authors wrote the paper together.

Funding: This research was funded by [the National Natural Science Foundation of China] grant number [61703280, 61603258], [the Science and Technology Planning Project of Shaoxing City of China] grant number [2017B70056, 2018C10013], [the General Research Project of Zhejiang Provincial Department of Education] grant number [Y201839944], and [the Public Welfare Technology Research Project of Zhejiang Province] grant number [LGG19F020007].

Conflicts of Interest: The author declares no conflict of interest.

\section{References}

1. Zadeh, L.A. Fuzzy sets. Inf. Control 1965, 8, 338-356. [CrossRef]

2. Atanassov, K.T. Intuitionistic fuzzy sets. Fuzzy Sets Syst. 1986, 20, 87-96. [CrossRef]

3. Chen, T.Y.; Han, Y.; Liao, S.Z. The largest sub-algebra and its generalized tautology in disturbing fuzzy propositional logic. Chin. J. Eng. Math. 2003, 20, 118-120.

4. Zadeh, L.A. The concept of a linguistic variable and its application to approximate reasoning-I. Inf. Sci. 1975, 8, 199-249. [CrossRef]

5. Smarandache, F. Neutrosophy: Neutrosophic Probability, Set, and Logic; American Research Press: Rehoboth, MA, USA, 1998.

6. Wang, H.; Smarandache, F.; Zhang, Y.Q.; Sunderraman, R. Single valued neutrosophic sets. Rev. Air Acad. 2010, 17, 1-10.

7. Wang, H.; Smarandache, F.; Zhang, Y.Q.; Sunderraman, R. Interval neutrosophic sets and logic: Theory and applications in computing. Int. Conf. Comput. Sci. 2006, 3991, 920-923.

8. Smarandache, F. n-Valued Refined Neutrosophic Logic and Its Applications in Physics. Prog. Phys. 2013, 4, 143-146.

9. Zhang, W.R. Bipolar fuzzy sets and relations: A computational framework for cognitive modeling and multiagent decision analysis. In Proceedings of the The Workshop on Fuzzy Information Processing Society Bi Conference, San Antonio, TX, USA, 18-21 December 1994; pp. 305-309.

10. Zhang, W.R. Yin Yang Bipolar Relativity: A Unifying Theory of Nature, Agents and Causality with Applications in Quantum Computing, Cognitive Informatics and Life Sciences; IGI Global: Hershey, PA, USA, 2011.

11. Mesiarova-Zemankova, A.; Ahmad, K. Extended multi-polarity and multi-polar-valued fuzzy sets. Fuzzy Sets Syst. 2014, 234, 61-78. [CrossRef]

12. Chen, J.J.; Li, S.G.; Ma, S.Q.; Wang, X.P. m-polar fuzzy sets: An extension of bipolar fuzzy sets. Sci. World J. 2014, 3, 1-8.

13. Bosc, P.; Pivert, O. On a fuzzy bipolar relational algebra. Inf. Sci. 2013, 219, 1-16. [CrossRef]

14. Manemaran, S.V.; Chellappa, B. Structures on bipolar fuzzy groups and bipolar fuzzy ideals under (T, S) Norms. Int. J. Comput. Appl. 2012, 9, 24-28. [CrossRef]

15. Zhou, M.; Li, S. Application of bipolar fuzzy sets in semirings. J. Math. Res. Appl. 2014, 34, 61-72.

16. Deli, I.; Ali, M.; Smarandache, F. Bipolar neutrosophic sets and their application based on multi-criteria decision making problems. In Proceedings of the International Conference on Advanced Mechatronic Systems, Beijing, China, 22-24 August 2015; pp. 249-254.

17. Pramanik, S.; Dey, P.P.; Giri, B.C.; Smarandache, F. Bipolar neutrosophic projection based models for solving multi-attribute decision making problems. Neutrosophic Sets Syst. 2017, 15, 70-79. 
18. Dey, P.P.; Pramanik, S.; Giri, B.C. TOPSIS for Solving Multi-Attribute Decision Making Problems under Bi-Polar Neutrosophic Environment. New Trends in Neutrosophic Theory and Application, Pons asbl; European Union: Brussels, Belgium, 2016; pp. 65-77.

19. Uluçay, V.; Deli, I.; Şahin, M. Similarity measures of bipolar neutrosophic sets and their application to multiple criteria decision making. Neural Comput. Appl. 2016, 29, 739-748. [CrossRef]

20. Wang, L.; Zhang, H.Y.; Wang, J.Q. Frank Choquet Bonferroni mean operators of bipolar neutrosophic sets and their application to multi-criteria decision-making problems. Int. J. Fuzzy Syst. 2017, 3, 1-16. [CrossRef]

21. Yu, D.J.; Wu, Y.Y. Interval-valued intuitionistic fuzzy Heronian mean operators and their application in multi-criteria decision making. Afr. J. Bus. Manag. 2012, 6, 4158-4168. [CrossRef]

22. Liu, P.D. The Evaluation Method and Application Research of Enterprise Information Level Based on Fuzzy Multi-Attribute Decision-Making; Beijing Jiaotong University: Bejing, China, 2010; pp. 113-129.

23. Pouresmaeil, H.; Shivanian, E.; Khorram, E.; Fathabadi, H.S. An Extended Method Using Topsis And Vikor For Multiple Attribute Decision Making with Multiple Decision Makers And Single Valued Neutrosophic Numbers. Adv. Appl. Stat. 2017, 50, 261-292. [CrossRef]

24. Zavadskas, E.K.; Bausys, R.; Lazauskas, M. Sustainable assessment of alternative sites for the construction of a waste incineration plant by applying WASPAS method with single-valued neutrosophic set. Sustainability 2015, 7, 15923-15936. [CrossRef]

25. Zavadskas, E.K.; Bausys, R.; Kaklauskas, A.; Ubartė, I.; Kuzminskè, A.; Gudienė, N. Sustainable market valuation of buildings by the single-valued neutrosophic MAMVA method. Appl. Soft Comput. 2017, 57, 74-87. [CrossRef]

26. Stanujkic, D.; Zavadskas, E.K.; Smarandache, F.; Brauers, W.K.M.; Karabasevic, D. A neutrosophic extension of the MULTIMOORA method. Informatica 2017, 28, 181-192. [CrossRef]

27. Li, Y.; Liu, P.; Chen, Y. Some Single Valued Neutrosophic Number Heronian Mean Operators and Their Application in Multiple Attribute Group Decision Making. Informatica 2016, 27, 85-110. [CrossRef]

28. Zavadskas, E.K.; Bausys, R.; Juodagalviene, B.; Garnyte-Sapranaviciene, I. Model for residential house element and material selection by neutrosophic MULTIMOORA method. Eng. Appl. Artif. Intell. 2017, 64, 315-324. [CrossRef] 